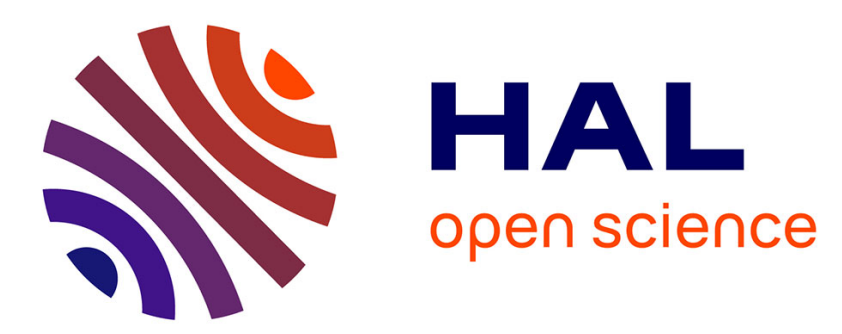

\title{
Electrical stimulation of developmental forces reveals the mechanism of limb formation in vertebrate embryos
}

Ameya Vaishnavi Murukutla, Vincent Fleury

\section{To cite this version:}

Ameya Vaishnavi Murukutla, Vincent Fleury. Electrical stimulation of developmental forces reveals the mechanism of limb formation in vertebrate embryos. European Physical Journal E: Soft matter and biological physics, 2019, 42 (8), 10.1140/epje/i2019-11869-8 . hal-02412420

\section{HAL Id: hal-02412420 \\ https://hal.science/hal-02412420}

Submitted on 15 Dec 2019

HAL is a multi-disciplinary open access archive for the deposit and dissemination of scientific research documents, whether they are published or not. The documents may come from teaching and research institutions in France or abroad, or from public or private research centers.
L'archive ouverte pluridisciplinaire HAL, est destinée au dépôt et à la diffusion de documents scientifiques de niveau recherche, publiés ou non, émanant des établissements d'enseignement et de recherche français ou étrangers, des laboratoires publics ou privés. 


\title{
Electrical stimulation of developmental forces reveals the mechanism of limb formation in vertebrate embryos
}

\author{
Vincent Fleury ${ }^{1}$ and Ameya Vaishnavi Murukutla \\ Laboratoire Matière et Systèmes Complexes \\ Université Paris Diderot/ UMR7057 CNRS \\ 10 rue Alice Domont et Léonie Duquet \\ 75013 Paris, France
}

\begin{abstract}
Current knowledge on limbs development lacks a physical description of the forces leading to formation of the limbs precursors or "buds". Earlier stages of development are driven by large scale morphogenetic movements, such as dipolar vortical flows and mechanical buckling, pulled by rings of cells. It is a natural hypothesis that similar phenomena occur during limb formation. However it is difficult to experiment on the developmental forces, in such a complex dynamic system. Here, we report a physical study of hindlimb bud formation in the chicken embryo. We use electrical stimulation to enhance the physical forces present in the tissue, prior to limb bud formation. By triggering the physical forces in a rapid and amplified pattern, we reveal the mechanism of formation of the hindlimbs : the early presumptive embryonic territory is composed of a set of rings encased like russian-dolls. Each ring constricts in an excitable pattern of force, and the limb buds are generated by folding at a pre-existing boundary between two rings, forming the dorsal and ventral ectoderms. The amniotic sac buckles at another boundary. Physiologically, the actuator of the excitable force is the tail bud pushing posteriorly along the median axis. The developmental dynamics suggests how animals may evolve by modification of the magnitude of these forces, within a common broken symmetry. On a practical level, localized electrical stimulation of morphogenetic forces opens the way to in vivo electrical engineering of tissues.
\end{abstract}

\section{Introduction.}

Understanding limb development and regeneration [1,2] is an important scientific endeavour. Molecular mechanisms of limb formation and their corresponding biochemical pathways have been studied extensively [3-5] and it has been shown that limbs can be generated by implanting growth factors soaked beads along the dorso-ventral boundary of embryo flanks [1]. However, physical forces that drive the positioning of limbs during morphogenesis remain elusive. Recently, a lot of work has been dedicated to the physics of living tissues.

\footnotetext{
${ }^{1}$ Author for correspondence, email : vincent.fleury@univ-paris-diderot.fr
} 
Physical principles of condensed soft-matter have been invoked at all biological scales, from intracellular $[6,7]$ to cellular scales $[8,9]$, from tissue $[10,11]$, to organ $[12,13]$ and even at the whole organism level [14]. The interaction between mechanical stresses, genetic expression and morphogenetic effects has been illustrated in various systems such as fly embryogenesis [15,16], lung [17] and vascular morphogenesis [18]. The correlation between tissue stiffness and cell behaviour or differentiation has been investigated by many, notably Discher et al. [19] and Asnacios et al. [20].

Large scale tissue movements such as dipolar vortices $[14,21]$ and tissue buckling $[22$, 23 ] occur during vertebrate embryonic development. The body form is the resulting integral of all deformation rates since the beginning of the embryogenic movements. In vertebrates, all these movements contribute, step-by-step, to positioning all organs including the limbs [24, 25].

Tissue movement in amniotes is driven by rings or "belts" of cells behaving like "purse strings" [14, 26, 27]. Similar supra-cellular "purse strings" have been observed in morphogenesis of nematode worms and flies and also in wound healing [27, 28]. As stated by Kiehart in as early as 1999 (Ref. 28, p. R604), "contractile purse strings are common place in both development and tissue homeostasis". This statement is particularly important with regard to the development of vertebrates.

Indeed, it has been shown that the reference configuration or "starting point" for the physical formation of the vertebrate embryo is a set of encased rings of cells present at the blastula stage (or flat blastodiscs for chicken embryos). They are composed of smaller cell sizes internally [22], and larger cells peripherally, Supp. Material. Fig. S1. The ring structure of the early embryo is already visible at the morula stage (first cell cleavages) [29]. The formation of this ring structure is due to a gradient of cell cycle period [30]. Since the geometry of the embryo at this stage is in rings and developmental forces are exerted by its cells, the forces also are exerted in a ring-like pattern [14, 21, 23, 26]. This is the origin of several almost circular "purse-strings" which deform and shape the early vertebrate embryo. Observation of developmental processes suggests that morphogenesis repeatedly uses the same mechanism for pattern formation : several contractions of "purse-strings" of excitable cells positioned initially in concentric domains. This provides an account for animal construction in which several contractions are triggered sequentially by reflex until an animal is formed.

However, although the initial ovocyte is round, the developing vertebrate embryo is not circular. This is because the first circular pull is by the edge, and it re-circulates around a 
stagnation point (Video 1, Fig. 1a) [14]. The flow around the stagnation point transposes the dynamics along the median axis (Video 2, Fig. 1b) [21, 22]. Thus, the embryo continues its morphogenesis by extending along the antero-posterior axis, forming a bilaterian animal. During this antero-posterior extension along the median axis, the lateral areas of the embryonic tissue are dragged and rotated in a vortical fashion (Video 2, Fig. 1c) [22, 24].

As a result of this drag, the initially circular boundaries acquire a typical bilateral guitarlike shape composed of encased deformed "rings", instead of circular rings, (Ref. 22. Fig. 2). The embryo rolls up as it folds. These folds occur systematically at the edges between two subsequent "guitar-like rings" [22]. The process is very robust, and transforms a nearly two dimensional pre-pattern of rings into a set of encased tubes (neural tube, gut, body, amniotic sac, Supp. Fig. 2). An important dynamic feature is that the tension force in the embryo is excitable [23]. We show in this article that the tension forces acting between rings are at all stages excitable, and that there exists a previously overlooked ring of cells along the body edge, which also constricts. The ring contracting along the body edge folds the presumptive limb tissue along the Dorso-Ventral (DV) boundary. This localizes the physical protrusions which are recognized as "limb buds", with a distinct dorsal skin and a ventral skin.

More specifically : in a first series of observations we study the movements and cell patterns during physiological formation of the limb buds by Time-Lapse (TL) and by Scanning Electron Microscopy (SEM). Then, we electrically stimulate the embryonic tissue before and during the moment of formation of the limb buds, to reveal the pattern of forces. The purpose is both to have a larger value of the force, and a more rapid expression of it. This increases the signal/noise ratio, and avoids mixing different effects occurring on a longer time scale such as cell migration or viscous dissipation. We conclude that the embryogenetic mechanism is a physical tensional process intrinsic to a set of contractile encased rings whose contraction is triggered in sequence.

\section{Materials and Methods}

\subsection{Sample preparation}

Here we describe the preparation of embryos for Time-Lapse imaging. This set up allows one to incubate embryos with cellular resolution for up to 15 hours (a statistics over 10 samples gives an interval between 6 and 15 hours, with an average of 10 hours). Generally, there is a phase of "recovery" after preparation of the embryo of approx. $1 / 2$ hour, and a 
variable phase of embryo fatigue before complete developmental arrest (we ascribe terminal embryo death to photo-bleaching).

\section{1.a Preparation of the incubation chamber}

The incubation chamber consists of a Petri dish cover (Duroplan, $10 \mathrm{~cm}$ ). A plastic ring of diameter $10 \mathrm{~cm}$, height $4 \mathrm{~mm}$ and width $1 \mathrm{~cm}$ is glued on the Petri dish cover to serve as edges of the incubation chamber (see Supplementary Figs. S3 and S4).

\section{1.b Preparation of a substrate for incubation}

Unlike other protocols for chicken embryos incubation, we do not incubate embryos on a layer of artificial gel, but on a fresh vitelline membrane, resting on a layer of thick albumin. The fresh vitelline membrane is obtained in the following way (Supplementary Figs. S3 and S4). A "not incubated" or "as received" egg is cracked and opened in a Petri dish (Duroplan, diameter $7 \mathrm{~cm}$ ) (Fig. S3a). A round patch of vitelline membrane is cut off from the top of the egg (Fig. S3b). The disc of vitelline membrane, and the vitelline gel above it is carefully transferred to a Petri dish containing Phosphate Buffer Saline solution from Dubbelco (PBS) (Fig. S3c). Next all the yolk is gently removed with a pipette, and by rinsing it in several baths of PBS (Fig. S3d). When the vitelline membrane is washed, it is transferred to the incubation chamber, to be used as substrate for embryo incubation (Fig. S3e). The vitelline membrane and the thick albumin on top of it are transferred with a spoon towards the incubation chamber, by letting them float in a bath of PBS, they are turned upside-down, and positioned in the center of the incubation chamber inside an aluminum ring (diam. $5 \mathrm{~cm}$, height $1 \mathrm{~cm}$, Fig. S3e, f). By turning the patch upside down the vitelline membrane is now on top, and the thick albumin underneath. The embryo rests on the vitelline membrane, as it does physiologically, but now it will be directly visible. The aluminum ring is removable. The provisory bath of PBS with the vitelline membrane inside it will also be used to transfer the embryo.

\section{1.c Preparation of the embryos}

Eggs are received from the farm EARL Morizeau, located in Les Bruyères (Chartres) (breeder Hubbard, JA57 hen, I66 rooster, yielding type 657 chicks). Eggs are collected on day one, which means approx. day 1.5 after fertilization (the study of earlier developmental stages requires other techniques, since the egg has to be fetched in the oviduct [29]). Eggs may be 
kept at $13^{\circ} \mathrm{C}$ for up to 14 days, during which the development is arrested. Development starts when eggs are put to incubate back at $37^{\circ} \mathrm{C}$ in standard incubators. (As days pass by, care must be taken that embryo development is progressively delayed approx. by one hour per day of stay in the $13^{\circ}$ refrigerator, also mortality increases progressively. This renders experimental work at a strict developmental stage difficult to organize).

For our studies, the embryos are removed from the egg at the desired developmental time for the experiment. The embryo is prepared by cracking open an egg in a Petri dish $7 \mathrm{~cm}$ wide. Then, the vitelline membrane is cut off in a circle, taking care that the embryo rests roughly in the center (Fig. S4a). The embryo and its vitelline membrane are transferred to a Petri dish with a spoon. There, it is rinsed in PBS (Fig. S4b, the arrow points to the embryo), up to the point that it is very clear. The embryo is next detached with fine tweezers from the vitelline membrane, and transferred to the bath of PBS inside the aluminium ring (Fig. S4c). Next the embryo is gently positioned on top of the vitelline membrane patch and held immobile with tweezers (Fig. S4d). Then the PBS is slowly removed, so that the embryo and the vitelline membrane underneath sediment. As the substrate touches the bottom, it spreads, and the embryo is stretched radially spontaneously. It recovers a diameter comparable to the initial one. Once the embryo is laying flat, the aluminum ring is removed. The remaining PBS is dried off with a piece of paper, and a ring of albumin is spread all around the embryo (Fig. S4e). The albumin serves three purposes : it provides some nutrients by diffusion, it provides water, and it keeps the embryo almost immobile, except for transient capillary movements.

\section{1.d Sealing and positioning of the incubation chamber}

The albumin is also used as glue to seal the incubation chamber (Fig. S4f). The incubation chamber is brought to the microscope (Nikon Eclipse, upright microscope), or a binocular (Leica Macrofluo) and rapidly covered with a Minitüb heating stage (Fig. S5; the star in S5 shows the heating stage). The heating stage temperature is set to $38^{\circ}$. The plastic ring height $(4 \mathrm{~mm})$ is critical as it allows the passing of both the incubating chamber and the heating stage (thickness $1 \mathrm{~cm}$ ) under the Nikon microscope objective (which has a long working distance of $12 \mathrm{~mm}$ (Fig. S4b)). For the Macrofluo, the working distance is not an issue. The temperature setpoint of the incubation chamber $\left(38^{\circ}\right)$ was determined empirically by testing the entire set up with a thermocouple located at the presumptive position of the embryo. 


\subsection{Image acquisition}

Time-Lapse movies were acquired with a monochrome HD camera Stingray 201 from Allied Vision Technology, interfaced with AVT-Smartview software. Light is shone on the embryo with a 1600 LED lamp from Schott, the light is approached at $45^{\circ}$ with the flexible fiber, and through a copper slit $4 \mathrm{~mm}$ wide and $2 \mathrm{~mm}$ deep. The embryo is observed through a second slit. By manually adjusting the position of the slits one obtains a shadowgraphic contrast, which is sharp enough for image analysis (individual cells are resolved, although not segmented). Light is toxic for the embryos and the intensity is kept as small as possible, and always < graduation 3 of the lamp power source.

\subsection{PIV analysis}

The extraction of the displacements is done numerically with the "Tracker" module developped by Olivier Cardoso and Bérangère Abou in ImageJ plugin environment (by Wayne Rasband, from NIH). It uses correlation function between small domains in subsequent images to identify the movements. A custom macro in ImageJ software "Selectagrid" allows one to generate semi-automatically a rectangular grid of points for the analysis of the displacements (a rectangular Region of Interest is selected and the macro generates automatically the matrix of points for PIV). The software and the custom macro is available on the academic site of the corresponding author at the address : http://www.msc.univ-paris-diderot.fr/ vfleury/portailPIV.html, or upon request.

\subsection{Electric shocks}

An aluminium board $3 \mathrm{~cm}$ wide and $15 \mathrm{cms}$ long is prepared. One end of the board is folded at $45^{\circ}$ to form the approaching head of the set up. Two flexible insulated copper wires, (diameter $1 \mathrm{~mm}$ and $15 \mathrm{~cm}$ long) are scotch-taped along the aluminum board (Supp. Material Fig. S6). Two thin varnish coated copper wires (diam. $50 \mu \mathrm{m}$ ) are cut to form the end point of the electrodes. The thinner wires are welded at the ends of the flexible wires. They hang away at the end of the board by approx. $2 \mathrm{~cm}$. The electrodes are bent by hand to fix their interspace distance. Another set up with a fixed electrode interdistance was also used, but in practice it was more practical to have both the flexible electrodes independent of each other and to fix the distance between them ony approximately. This is required since the two electrodes will rarely be at the same height because of embryonic topography. Therefore it is important to have the two electrodes flexible and independent, otherwise there is a risk of perforating the embryo with one of the electrodes while approaching the other into contact on a locally 
slanting topography. The flexible wires are connected to a pulse generator from Hewlett Packard 8002A (Supp. Material Fig. S6). The maximum voltage used was $1.5 \mathrm{~V}$, and the maximum duration of the electric shock was 10 Seconds at $1 \mathrm{~Hz}$. It is a casual observation that electric shocks below $5 \mathrm{~V}$ are safe. We measured the current with a low resistance galvanometer in series (Fluke). The total current passing through the electrodes was found in the range 1-20 microAmpères. For a 10 Second / 1 Volt /10 microAmperes electric shock, the energy dissipated is $10^{-4} \mathrm{~J}$. Considering that the heat capacity of water is $\sim 4.2 \mathrm{~J}^{-\mathrm{g}^{-1}} \cdot \mathrm{K}^{-1}$, and that the typical embryo volume located between the electrodes is $5 \mathrm{~mm} \times 5 \mathrm{mmx} 0.1 \mathrm{~mm}=2.5$ $\mathrm{mm}^{3}$ we estimate the average temperature increase $<0.1 \mathrm{~K}$. However, there is a higher energy density dissipated under the electrodes since they have a sharp tip. Therefore, we checked the potential necrotic effect of such electric shocks by Trypan Blue staining (a classical staining of dead cells, with a $1.4 \%$ solution from Sigma). We found only a very localized staining quite close to the electrodes (3 samples), of a few cells (Supp. Material Fig. S7); the control on a fresh sample shows no staining at all (data not shown). This localized, small, necrotic effect does not alter the interpretation of the global deformation fields which are observed (e. g., the deformation field observed in Supp. Material Fig. S7).

\section{Results}

\subsection{Embryo developmental dynamics.}

We observed movements in the embryo during tailbud and early stages of limb formation (HH stages 14-18 [34] ) using TL video-microscopy. The spatio-temporal analysis of movements in the tailbud region extracted from time-lapse Videos (Videos 3, 4, 5) of 3 embryos at the same stage are shown in Fig. 3. Firstly, we find that the anal area is compressed by the forming tail bud (Fig. 3a Left, see also Video 6 and 7 at a higher resolution), until a rapid constriction of the surrounding ring occurs (Fig. 3a Right). Fig. 3b shows similar results for two other embryos. Following this constriction, the anal area folds ventrally and the tail bud passes over the average plane of the embryo. While doing so it shears the lateral plates in a vortical fashion (Fig. 4a,b, Videos 8, 9). The contraction of the surrounding ring exerts a movement antagonist to the vortical shear, thus amplifying the folding of the tissue. In the ring of tissue surrounding the tail bud, the cells are oriented as shown in Fig. 4c.

The presumptive limb territory constricts along the presumptive edge of the limb. We show in Fig. 5a, b the spatio-temporal analysis of the dynamics of the limb edge, as extracted from 3 time-lapse Videos (Videos 10,11, 12) of embryos at the same stage. The limb edge 
appears obviously as the tissue continues to fold ventrally. Fig. $5 \mathrm{~b}$ shows the formation of the limb fold and the propagation of the limb edge. (We also observe that the contact of the tail bud, with the amniotic ring, triggers the formation of the amniotic sac, Videos 4, 11 and 12). The folding wave of the limbs progresses following the presumptive DV boundary where the Apical Ectodermal Ridge (AER) appears [1-6]. The limb precursor is still surrounded by a belt of aligned cells (star in Fig. 5b Bottom, see also Fig. 4c).

We confirm the structure of encased rings during this process by SEM. Fig. 6 shows observations of the tissue in the anal area, close to the presumptive hindlimb territory before the tail bud hangs post-anally (Fig. 6a), and just afterwards (Fig. 6b). We clearly see the DV boundary, with cells having different sizes, and different density of the Extra-Cellular Matrix (ECM; we leave it as an open question, whether the increased density of the ECM is a mere consequence of increased cell density, or is related to genuine cell differentiation). We also see two encased rings or "belts" of aligned cells surrounding the presumptive limb territory.

Since the posterior push of the tail triggers ring contraction, one may wonder what is the actual mechanism of tail elongation? We observe a caudal flow of cells emanating from the tail-bud and flowing anteriorly (Fig. 7a). This flow induces an anterior advective flow of the entire somitic tissue (Fig. 7b, Video 13). The cell population pressure in the tail bud explains naturally both the anterograde flow of the somites and the retrograde movement of the tail by the principle of action and reaction (stated otherwise, the source of cell flow in the tail is a place of high pressure). It is a classical result that the tail bud is a singular area where growth factors, especially FGF8, are localized, see Ref. 31, especially stages 13 to 15.

\subsection{Electric stimulation of embryo developmental dynamics.}

In order to analyze the observed contractions, we stimulated the embryo with thin copper electrodes (diam. $50 \mu \mathrm{m}$ ), connected to the pulse generator. The applied potential was in the range $[1 \mathrm{~V}-1.5 \mathrm{~V}]$. The success rate of the experiment was $100 \%$. In all cases, rapid electric shocks $(1 \mathrm{~Hz}$ for $\mathrm{t}<10 \mathrm{Sec}$., often $3 \mathrm{Sec}$.) would elicit a contraction of the tissue, lasting over tens of minutes. We found that all tested zones on the embryo body (neural crests, body, tail bud, etc.) were sensitive to electric shocks, and responded by a contraction. However, as we wish to relate the mechanism of limb formation to the repeated use of excitable contractions in the embryo, we apply electric stimuli on the embryo from the onset of morphogenesis, until limb formation. The effect of the stimuli tends to relax with a time constant $\sim 15$ min. 
We have shown [14], and it has been confirmed [26] that embryogenesis starts by a rotatory contraction in the posterior area of the blastodisc (the moment when the avian embryo is a round disc). Our first tests were performed on day 1 when contractile cells first appear to form a ring around the still circular blastula [14]. We incubated eggs for 15 hours, and applied shocks at the onset of gastrulation, while the peripheral ring constricts in the direction of the presumptive anus. We applied shocks in the area of the presumptive primitive streak where gastrulation starts in amniotes. We observed an increase in deformation rate 20X (Fig. 8a, Video 14).

We then worked on embryos at the onset of neurulation when the contour of the embryo extends in the posterior direction and the embryo tissue starts to be pulled posteriorly. We found that a small ( $\sim 3 \mathrm{Sec}$.) electric shock was able to trigger contraction in the embryo (Fig. 8b, Video 15). Electric shock applied in posterior area elicits a long range contraction along the median axis, pulling on the area of the presumptive chord.

Then we worked on embryos whose body axis extended into the presumptive hindlimb area. Around the presumptive limb territory localized shocks elicited contractions, amplified the predicted pattern of forces, and propagated to the entire deformed "rings". The pattern of the movement was found to follow tissue prepattern, i.e. the "guitar-like" contour, with a contraction oriented towards the anal area. Fig. 9a-e shows the result of electrical stimulation on the posterior territory at $\mathrm{HH} 14$ to 17 , i.e. from a stage with rudimentary tail bud up to the moment when the body rolls up and the limb bud forms (see Ref. 32 for the nomenclature of Hamilton and Hamburger stages $\mathrm{HH}$ ). All experiments show a rapid contraction in the anal area and the presumptive body contour. Fig. 9a shows the result of electrical stimulation on the anal area of an embryo at HH14 (Video 16). Fig. 9b shows the pattern of contraction obtained at HH15, when the contour of the presumptive tail bud is visible (Video 17). A stronger contraction oriented along the tissue boundaries is observed, with traction forces aligned with the presumptive limb boundary. Fig. 9c (Video 18) shows the rapid contraction obtained by electric stimulation when the tail bud just starts to hang over the anal territory, before formation of the limb edge. A massive contraction oriented around the hindlimb territory and pointing towards the anus is observed. Fig. 9d (Video 19) shows that a similar pattern is obtained when a contraction is triggered as the limb edge is starting to form. Fig. 9e (Videos 20) shows the contraction when the limb edge is well visible in the posterior area. Stimulating the limb plate provokes a large contraction oriented ventrally and the contour of the embryo appears in a few minutes (complete roll up occurs in two hours instead of a day). 
The experiments (e.g. Videos 16 and 20 especially) show that the electric shock also triggers amnion formation, by the same mechanism.

Quantitatively, we can follow the morphogenesis for a few minutes prior to applying the electric shock and see experimentally how the deformation behaves as a function of time by following by PIV reference points. We chose points aligned in the direction of the larger visible contraction (principal stress direction, selected by direct observation). Everything else being equal, the change in deformation rate can only be ascribed to an increase in the magnitude of the force. Fig. 10a shows the evolution of the deformation between two selected points, for an electric shock applied on the chorionic fold (Video 21). A very conspicuous increase in deformation rate (slope of the curve) is obtained (of magnitude 9X), which is seen to decay with a relaxation time of 15 minutes. It was also noted that the mere contact with the electrode, prior to any electric shock, already caused a small increase in the developmental rate. Similarly, we follow the deformation rate when applying an electric shock close to the contour of the presumptive limb bud (Video 22), as the limb blade starts to form. We find a massive acceleration of the process, with a rapid formation of the edge of presumptive limb, including the AER. We note that the contralateral area of embryo folds much less, and a strong asymmetry becomes visible. This proves that the roll up is indeed localized and accelerated by the electric shock. In the embryo shown in Fig. 10b, as the first acceleration was only moderate $(\sim 5 \mathrm{X})$ we applied a second electric shock, to reach an increase in deformation rate of order $8 \mathrm{X}$. This shows that the experiment is flexible, and shocks can be iterated. The moment of the electric shocks is symbolized in the figures by a "lightning" symbol. Finally, Fig. 10c shows a detailed analysis of the orthoradial contraction, following an electric shock. It shows a continuity in movement but a discontinuity of the slope (white arrow), at the boundary between the ventral tissue and the presumptive limb tissue. The velocity of the tissue is $2 \mathrm{X}$ higher in the belt of tissue surrounding the limb precursors, which confirms that the surrounding belt of aligned cells contributes more to limb folding.

\section{Discussion}

The purpose of this work is to understand the mechanistic rationale behind formation of limbs in vertebrates. We assume that body formation is a global phenomenon amenable to a physical description. It is known that the starting point for embryo development is a round zygote (fertilized ovocyte). In the first cell cleavage rounds, the blastula acquires a structure with concentric rings of cells that are slightly skewed posteriorly (as a consequence of the 
sperm entry point). This is called radial cleavage in developmental biology. It has been shown that this radial cleavage is a fundamental pattern which is not evolved in its details but emerges globally [33]. It is known that this pattern is related to a gradient of fat across the blastula [34], fat being an inhibitor of the cell cleavage cycle. We have shown mathematically that repeated cell cleavage in a gradient of fat indeed produces concentric rings of cells with a stepwise variation of cell sizes [30]. Morphogenesis starts by movements induced by contraction of these rings. The first movements consist of rotatory contractions driven by the peripheral ring. This has been known experimentally since the seminal work of Wetzel [35] and understood as a vortical elasto-visco-plastic flow only recently [14]. We have previously shown that the embryo then folds in cascade along the circular lines separating cell types [22]. This dynamics has been confirmed recently [36]. In this article we show that early contractions can be excited electrically and large amplification factors can be obtained for these contractions. Hence, the contractility is present and latent from the very start of embryogenesis; the available force is actually much bigger than the one used for normal embryogenesis. It is well observed in biology that normal metabolism uses only a fraction of the maximum available force.

We then addressed the questions of body outline and limb formation. Observing the developmental process in detail by optical microscopy and SEM we observed these concentric rings constrict along the body edge and initiate the ventral folding of the embryo. This is followed by a dorsal folding of the amnion and separation of dorsal and ventral tissues by folding. We managed to stimulate these constricting forces locally. We obtained an increase in developmental rates of the order of $10 \mathrm{X}$ around the limb territory. The pattern of contraction follows the existing "rings", although at these stages these "rings" are more "guitar-like" than circular.

We observe that the force pattern is distributed along discrete rings and is triggered in cascade. Hence, in early embryos there exists a prepattern of cellular territories that form contractile rings encased like russian-dolls. These rings have different mechanical properties. This pattern of rings reflects the early cleavage pattern, and the mechanical properties are related to cell sizes. When constriction starts, a series of folds forms, starting with the neural tube, then the limb bud with its sharp edge and the body contour, and finally the amnion and chorion folds, as shown in Ref. 23. All folds form along ring boundaries. Tension acts at all moments during this process to provide the driving force. An actual supra-cellular "cable" is observed along the presumptive body edge (SEM photo $6 \mathrm{~b}$, a similar cable, also visible in $6 \mathrm{~b}$, 
was found along the amnion contour in Refs. 23 and 27, and along the blastodisc edge in Ref.26).

We have shown here that this driving force can be amplified by electric stimulation, similar to muscular contraction in adults [37]. The contraction of the rings transforms the 2D pattern into a 3D body, and the limb blade points outwards at the Dorso-Ventral boundary forming a "paddle" like shape since the embryo has a "guitar" like pattern at this stage. We have also shown that the pattern of contraction is asymmetrical when the force is exerted unilaterally. During the normal process, developmental dynamics mediated by FGF8 [31] extends the tail bud posteriorly. This pushes along the median axis and compresses and stimulates the anal area until a contraction rapidly folds the anal area ventrally and the process of limb bud formation starts symmetrically. This shows that the physical actuator of this contraction is the extending tail bud.

These experiments show that animal phenotypes will be dependent on the magnitude and temporal pattern of the ring contractions, within a global broken symmetry which is common to the vertebrates phylum. They also show that it is possible to control morphogenesis with electromechanical instruments.

We have shown here that electric stimulation can trigger the contractions. Preliminary results show that mechanical contact also triggers the contraction. It is at all possible that other physical or molecular facts may constitute triggering events (e.g. calcium waves, neural spiking etc.). The mechanism described here couples physical morphogenesis to bioelectric fields as described recently by Levine [38] From an evolutionary point of view, the fact that embryo morphogenesis is structured by a set of excitable rings makes the process very robust since the actual triggering event is not essential, and it may vary from one taxon to another. For example, in chicken and crocodile the initial excitation for amnion formation was shown to be different [23], but the final result is nevertheless identical. An important aspect of the results presented here is that, the morphogenesis of the animal can be viewed as a single process or phenomenon, since once a set of excitable rings exists, an initial triggering cue triggers the entire process in cascade.

This work also raises a physics question regarding the upscaling of molecular phenomena, up to the formation of global organisms, and especially animals. Indeed, it is a major difference between animals and plants that in animals cell division occurs by contraction of a molecular acto-myosin "purse string" which cleaves the cells, while in plants cell divison occurs by plane-wall polymerization, oriented by tensile stress [39]. Therefore, in animals, purse-string formation and contraction is primarily an active molecular phenomenon 
at the cell-scale. However, such internal strings propagate a supra-cellular orientational order forming larger rings of actin and myosin which upscales ring contraction. We have shown here, that in vertebrate embryos, these purse-strings simply stir and fold the blastula in a quadrupolar fashion. The end result, or "attractor", of these dynamic movements will be identified as an animal : a set of motile tubes which move and digest. Movement and digestion are, again, made possible by upscaled contractions of circumferential rings (identified now as muscles). As shown by the work in Ref. 40, this supra-cellular purse-string mechanism is already present in jellyfish : it is ancestral. The dynamics of purse-string contraction in jellyfish can be up to 600 times (!) faster than in other animals [40]. Since unicellular division, supracellular morphogenesis, tube movement and even digestion, are driven by similar active rings, albeit upscaled, it may be wondered how straightforward the ladder to climb, in the morphogenetic space, from protozoans to organized animals, might be. Finally, while the embryo has been described here qualitatively as an active visco-elastic material submitted to purse-string forces, it should be reminded that cellular reorganisations are also observed, especially cell intercalation [41], which have an impact on the specific constitutive equation and driving force pattern. Physically, intercalation contributes to amplify dipolar movements [26], since it occurs along physical lines of the embryo such as the median axis or neural folds. Intercalation by active "T1" shifts (oriented topologial rearrangements) is theoretically a source of shear force [42].

Ethics statement : These experiments are authorized by French law R214-87 modified by the Décret n2013-118.

Acknowledgements. One set of experiments was performed by undergraduate student Marc Niemet of Université Paris-Diderot. We thank Jean-Loup Duband, Nicolas Odartchenko and Nicolas Chevalier for their interest and support in this work. We thank anonymous referees for constructive remarks. SEM was performed at Institut Jacques Monod imaging facility.

Authors contribution. Both authors designed and performed in vivo Time-Lapse development experiments and their analysis. Vincent Fleury was more involved in the electric shocks part, and Ameya Murukutla more in the Scanning Electron Microscopy. Both authors contributed to writing the manuscript. 


\section{References}

1. M. J. Cohn, J. C., Izpisua-Belmonte, H. Abud, J. K. Heath, C. Tickle, Cell, 80 (5), 73946, (1995).

2. H. V. Tanaka, N. Ch. Y. Ng, Z. Y. Yu, M. M. Casco-Robles, F. Mauro, P. A. Tsons, C. Chiba, Nature Comm., 7, 11069, (2016).

3. R. L. Johnson and C. Tabin, Cell, 90, 979-990 (1997).

4. M. Altabef, J. D. Clarke, C. Tickle, Development, 124, (22) 4547-4556 (1997).

5. J. C. Casanova, V. Uribe, C. Badia-Careaga, G. Giovinazzo, M. Torres, J. J. SanzEzquerro, Development 138, 1195-1205 (2011).

6. F. J. Nedelec, T. Surrey, A. C. Maggs, S. Leibler, Nature 389, 305-308 (1997).

7. J. Lammerding, Comp. Physiol. 1(2), 783-807 (2011).

8. P. A. Janmey, C. A. McCulloch, Annu. Rev. Biomed. Eng. 9, 1-34 (2007).

9. N. Bufi, P. Durand-Smet, A. Asnacios, Single-cell mechanics: The parallel plates technique, in Biophysical Methods in Cell Biology, Chapter 11, (Elsevier, Amsterdam, 2015).

10. F. Graner, J. A. Glazier, Phys. Rev. Lett. 69, 2013-2016 (1992).

11. G. W. Brodland, D. I.-L. Chen, J. H. Veldhuis, Int. J. of Plasticity 22, 965-995 (2006).

12. M. Unbekandt, M., P. M. del Moral, F. G. Sala, S. Bellusci, D. Warburton, D., V. Fleury, Mechanisms of Development 125 (3-4), 314-324 (2008).

13. T. Savin, N. A. Kurpios, A. E. Shyer, P. Florescu, H. Liang, L. Mahadevan L., C. J. Tabin, Nature 476, 57-62 (2011).

14. V. Fleury, Organogenesis 2 (1), 6-16 (2005).

15. E. Farge, Current Biology 13, 1365-1377 (2003).

16. P.-L. Bardet, B. Guirao, C. Paoletti, F. Serman, V. Léopold, F. Bosveld, Y. Goya, V. Mirouse, F. Graner, Y. Bellaïche, Dev. Cell 25, 534-546 (2013).

17. Z. Tang, Y. Hu, Z. Wang, K. Jiang, C. Zhan, W. F. Marshall, N. Tang, Developmental cell 44, 13-325 (2018).

18. F. Le Noble, D. Moyon, L. Pardanaud, L. Yuan, V. Djonov, R. Mattheijssen, C. Bréant, V. Fleury, A. Eichmann, Development 131, 361-375, (2004).

19. A. J. Engler, S. Sen, H. L. Sweeney and D. E. Discher, Cell 126, 677-689, (2006). 
20. D. Mitrossilis, J. Fouchard, D. Pereira, F. Postic, A. Richert, M. Saint-Jean, and A. Asnacios, PNAS 107 (38), 16518-16523 (2010).

21. V. Fleury, Biosystems, Special issue "Morphogenesis", 109, 460-474 (2012).

22. V. Fleury, N. Chevalier, F. Furfaro, J.-L. Duband, Eu. Phys. J. E 38, (6), 1-19 (2015).

23. V. Fleury, A. V. Murukutla, A., N. Chevalier, B. Gallois, B., M. Capellazzi-Resta, P. Picquet, A. Peaucelle, Phys. Rev. E 94, 022426-022444 (2016).

24. O. P. Boryskina, A. Al-Kilani, V. Fleury, Eu. Phys. J. App. Phys. 55 (2), 21101$21112(2011)$.

25. V. Fleury, O. P Boryskina, A. Al-Kilani, C. R. Acad. Sci. Biol. 334 (7), 505-15 (2011).

26. E. Rozbicki, M. Chuai, A.I. Karjalainen, F. Song, H. M. Sang, R. Martin, H. J. Knölker, M. P. MacDonald, C. J. Weijer, Nat. Cell. Biol 17, 397-408, (2015).

27. N. Tipping, D. Wilson, Anat. Rec. (Hoboken) 294 (7), 1143-9 (2011).

28. D. P. Kiehart, Current Biology 9, R602-R605 (1999).

29. H. C. Lee, H. J. Choi, T. S. Park, S. I. Lee, Y. M. Kim, S. Rengaraj, H. Nagai, G. Sheng, J. M. Lim, J. Y. Han, PLoS ONE 8 (11), e80631, (2016).

30. V. Fleury, Chaos, Solitons and Fractals, 105, 230-234, (2017).

31 T. Mikawa, A. Poh, K. Kelly, Y. Ishii, D. Reese, Dev Dyn. 229 (3), 422-32 (2004).

32. V. Hamburger, H. L. Hamilton, Journal of Morphology 88 (1), 49-92 (1951).

33. J. W. Valentine, PNAS, 94 (15), 8001-8005 (1997).

34. E. B. Wilson, The Cell and development in heredity, Third edition (Macmillan, New York, 1928).

35. R.Wetzel, Uber den primitivknotten des Hühnchens, Vehr. physik.-med. Ges. Würzburg 40, H.5 (1929).

36. R. Asai, Y. Haneda, D. Seya, Y. Arima, K. Fukda, Y. Kurihara, S. Miyagawa-Tomita, H. Kurihara, Sci Rep. 7, 8955 1-13 (2017).

37. M. Piccolino, Trends in neurosciences, 20 (10), 443-448 (1997).

38. M. Levine, Mol. Biol. Cell, 25 (24), 835-850 (2014).

39. M. Louveaux, J. D. Julien, V. Mirabe, A. Boudaoud, O. Hamant, PNAS, 26, 113 (30), E4294-303 (2016).

40. Z. Kamran, K. Zellner, H. Kyriazes, C. M. Kraus, J.-B. Reynier, and J. E. Malamy, BMC Dev. Biol. 17, 17 (2017).

41.R. Keller, L. Davidson, A. Edlund, T. Elul, M. Ezin, D. Shook, P. Skoglund, Philos. Trans. R. Soc. Lond. B Biol. Sci. 355 (1399), 897-922 (2000). 
42. M. Popovic A. Nandi, M. Merkel, R. Etournay, S. Eaton, F. Jülicher and G. Salbreux, New Journal of Physics 19033006 (2017). 
Figure Legends

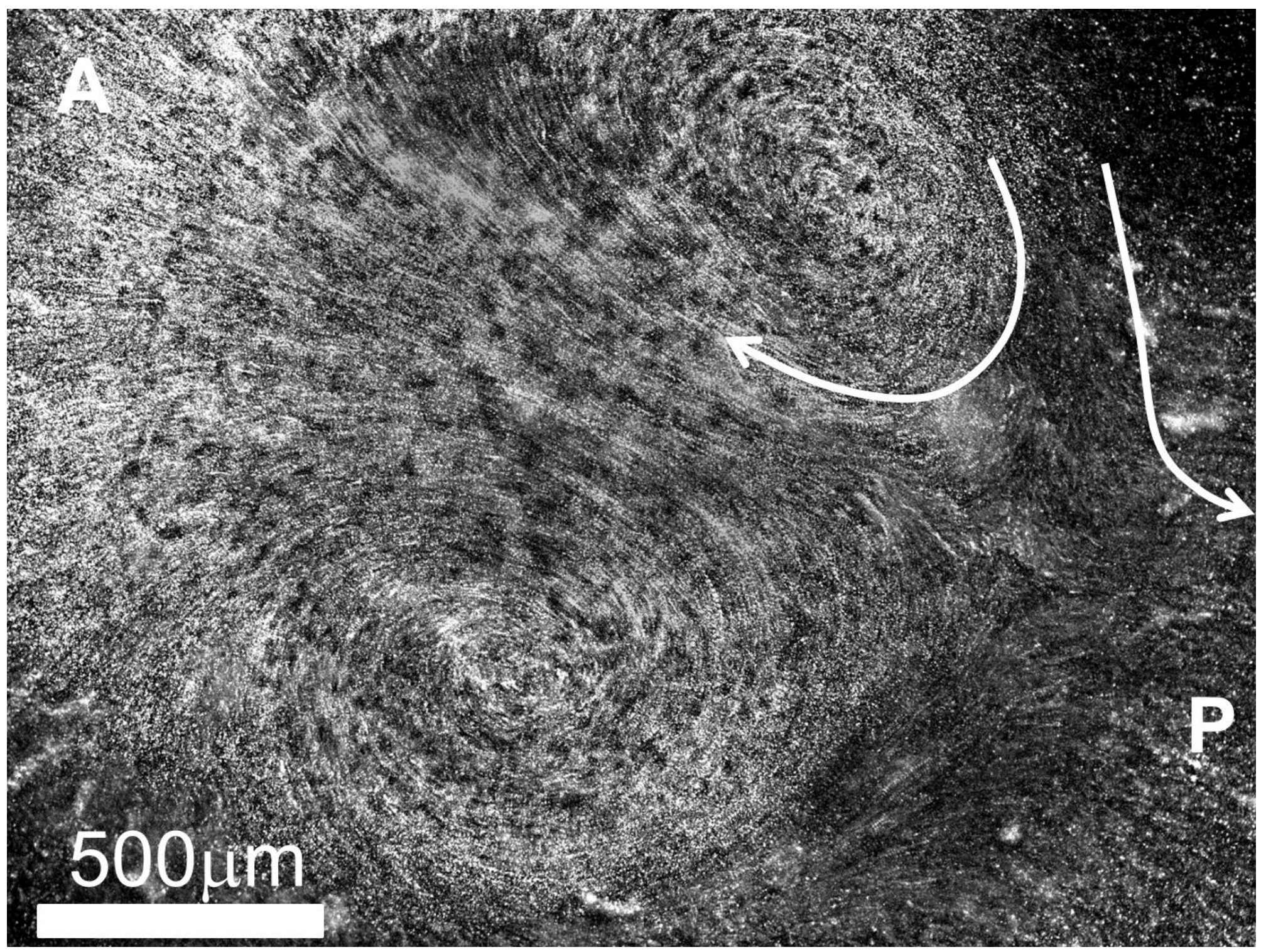

Figure 1a 


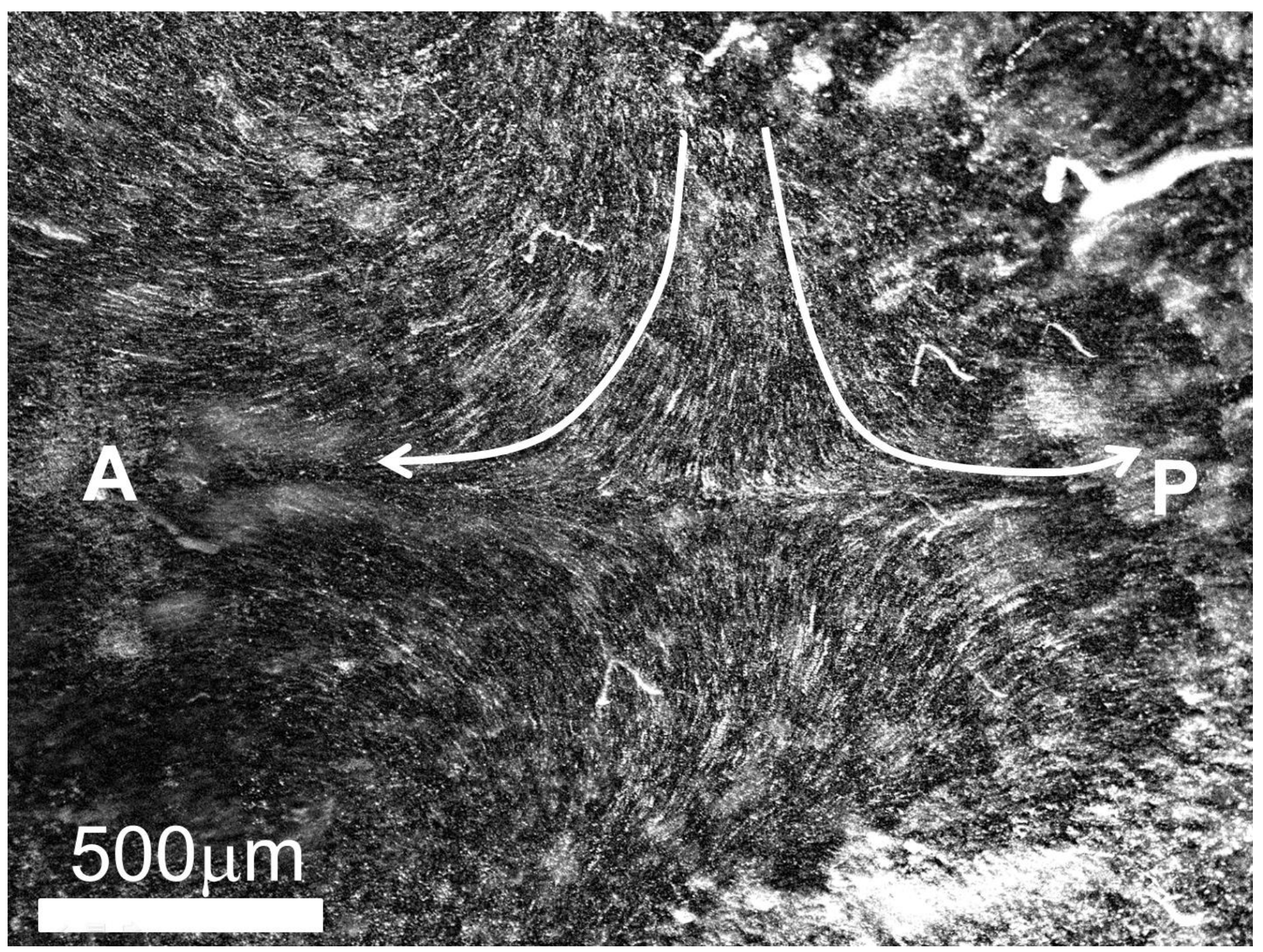

Figure 1b 


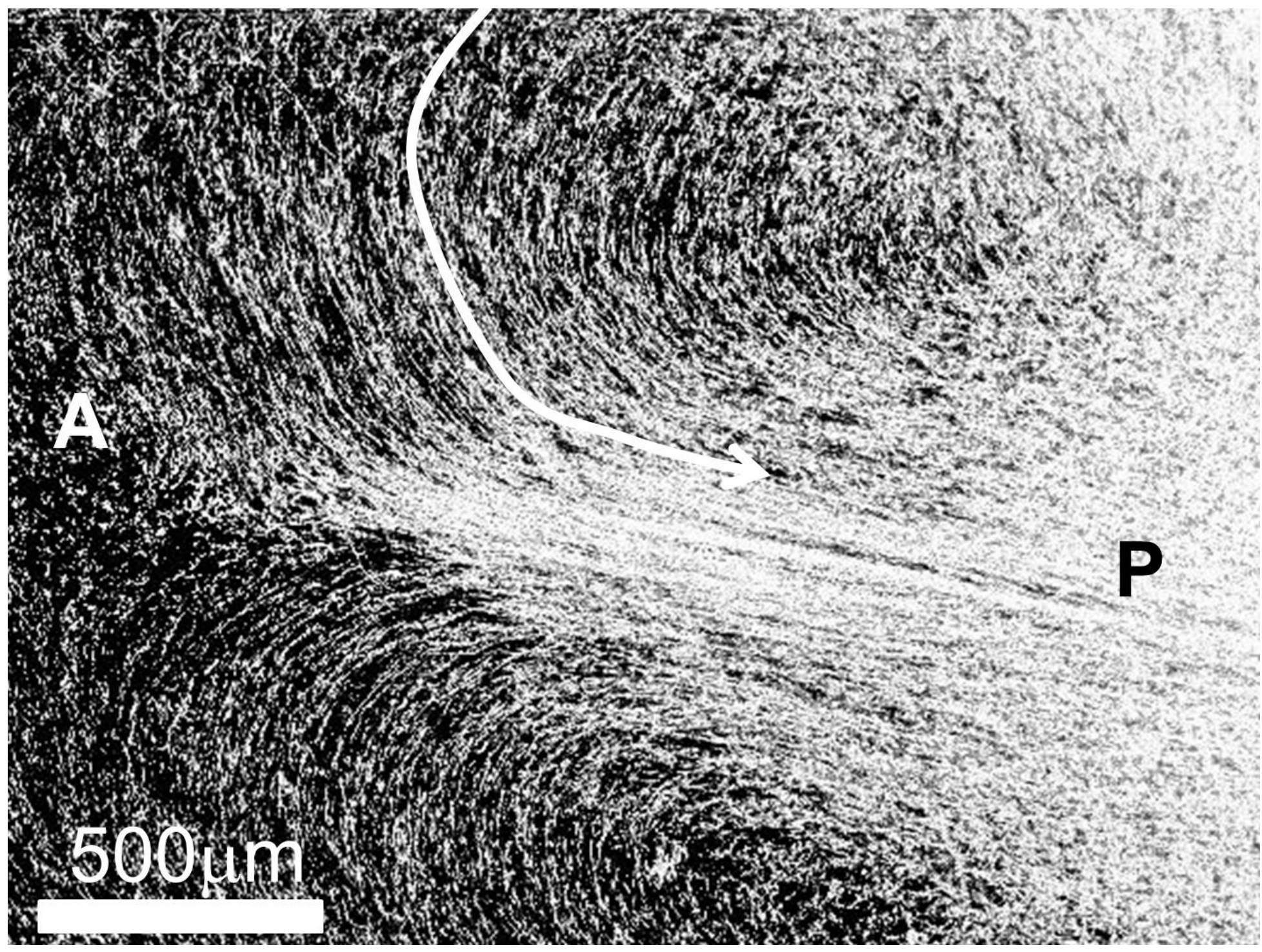

Figure 1c

Figure 1. Title : Early visco-elastic flows in the blastula. The «reference configuration » for the formation of vertebrate embryos is a round blastula. The blastula undergoes first a pull by the posterior edge which generates large visco-elastic vortices (Fig. 1a Mag. 4X, from Video 1). Around the stagnation point, the contractile area is transposed along the Antero-Posterior axis (Fig. 1b Mag. 4X, Video 2). Finally, the blastula undergoes a posterior traction, also formed of large vortices (Fig. 1c Mag 4X. from Video 2). The flow lines are generated by superimposing approx. 60 minutes of flow observed in shadowgraph, with a time interval of 1 minute. The white spots correspond generally to the nuclei of cells which are denser optically and diffuse light more. The grayscale is thresholded to extract the movements of the cells over the background. 


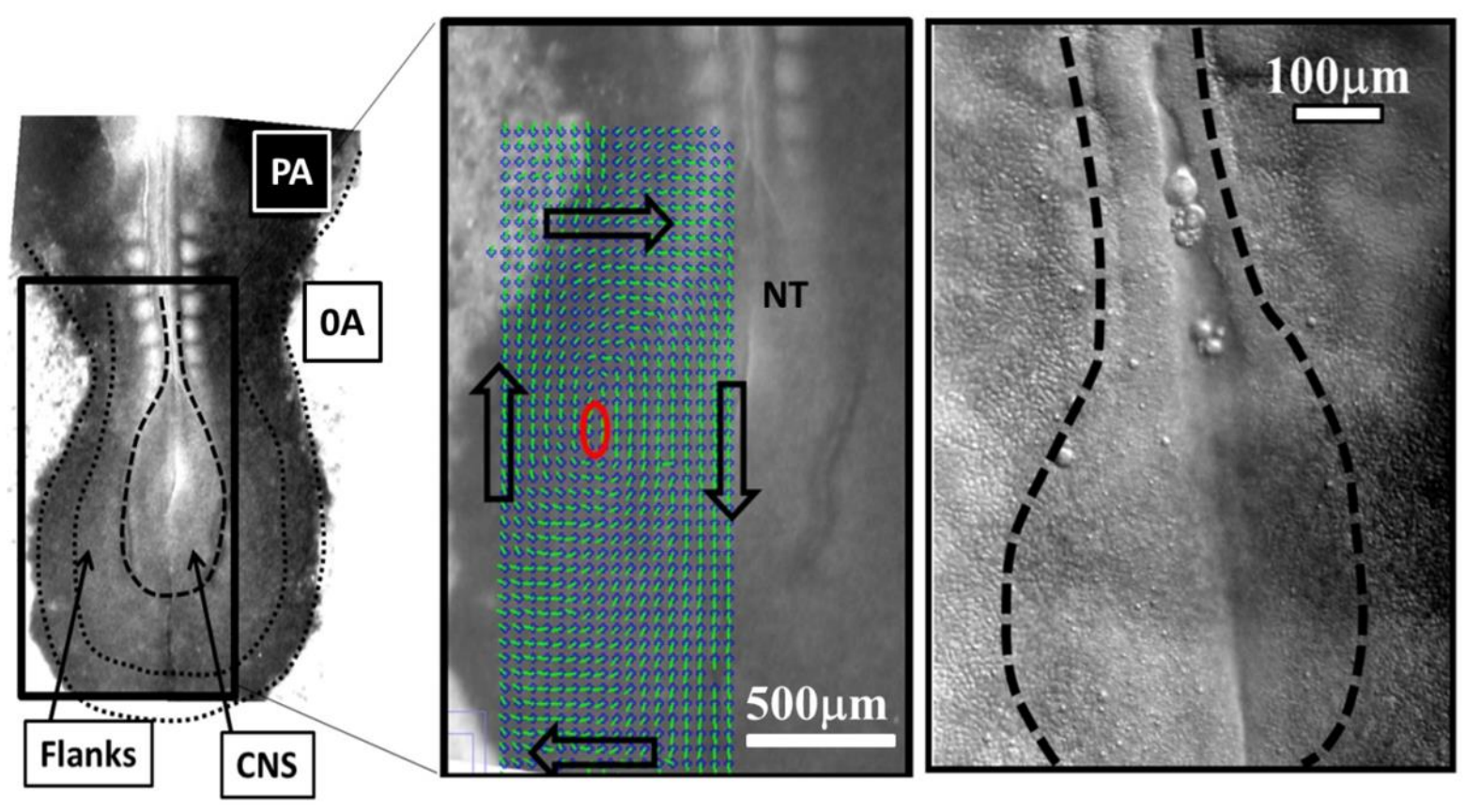

Figure 2

Figure 2 Title : Fold formation. Reprinted and adapted from Ref. 22. During extension of the body axis, the embryo folds in a process called "neurulation". The folds propagate while the tissue rotates. Fig. 2-Left : the image shows the structure acquired progressively by the embryo : the embryo is composed of encased "guitar-like" contours, forming the central nervous system (CNS), the flanks, the extra-embryonic organs; (OA : Opaque Area, PA Pellucid Area). Fig. 2-Middle: as the neural tube (NT) and the presumptive tail tissue extend, the lateral plate rotates in a vortical fashion; the image shows the PIV analysis of the movements showing a dipolar vortical flow. This movement deforms the boundaries between the embryo compartments. Fig. 2-Right, a magnified view of the boundary of the presumptive neural system shows how the embryo compartments form: the embryo folds exactly along the line separating smaller and larger cells; this line is continuously deformed by the tissue flow. 

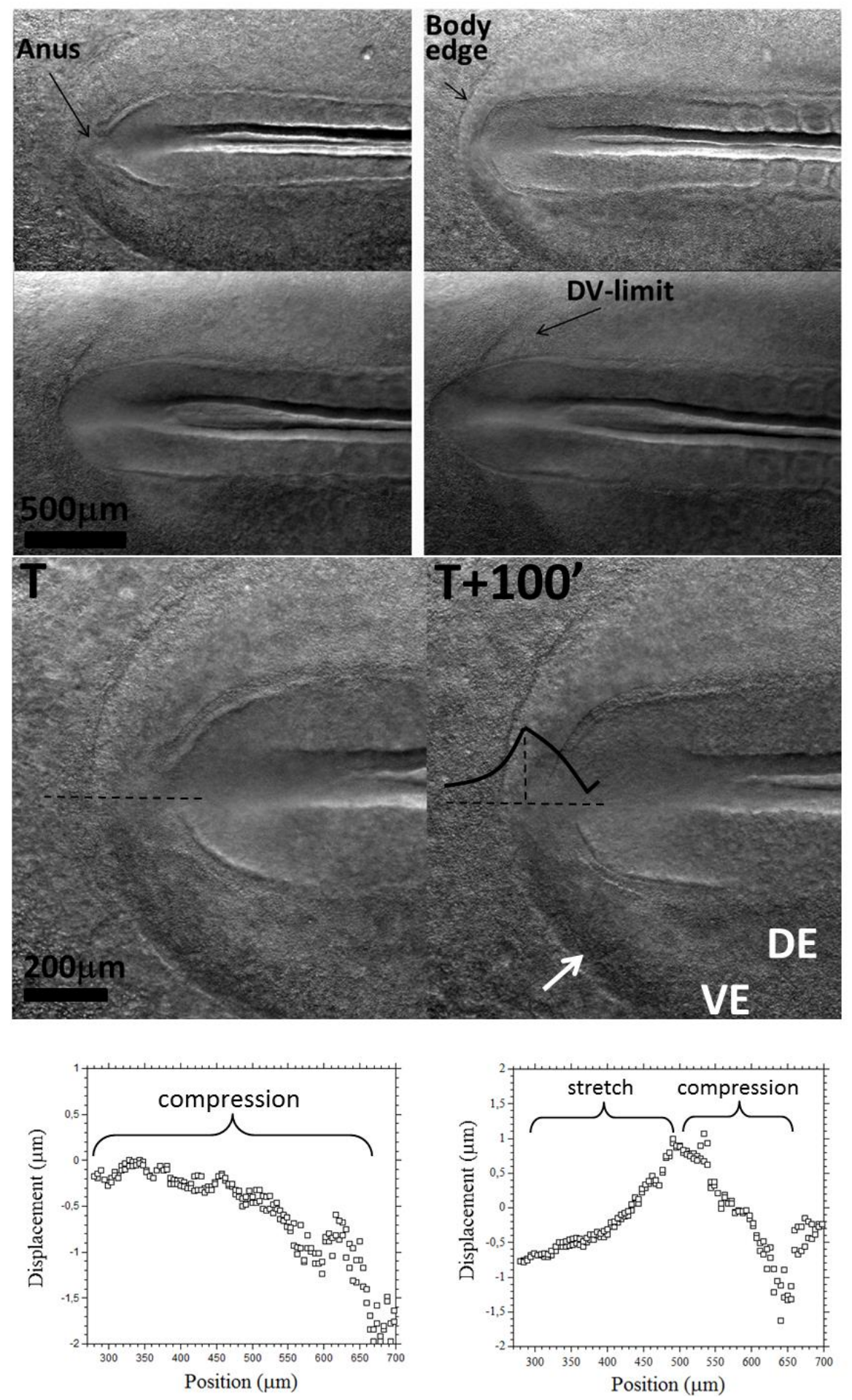

Figure 3a 

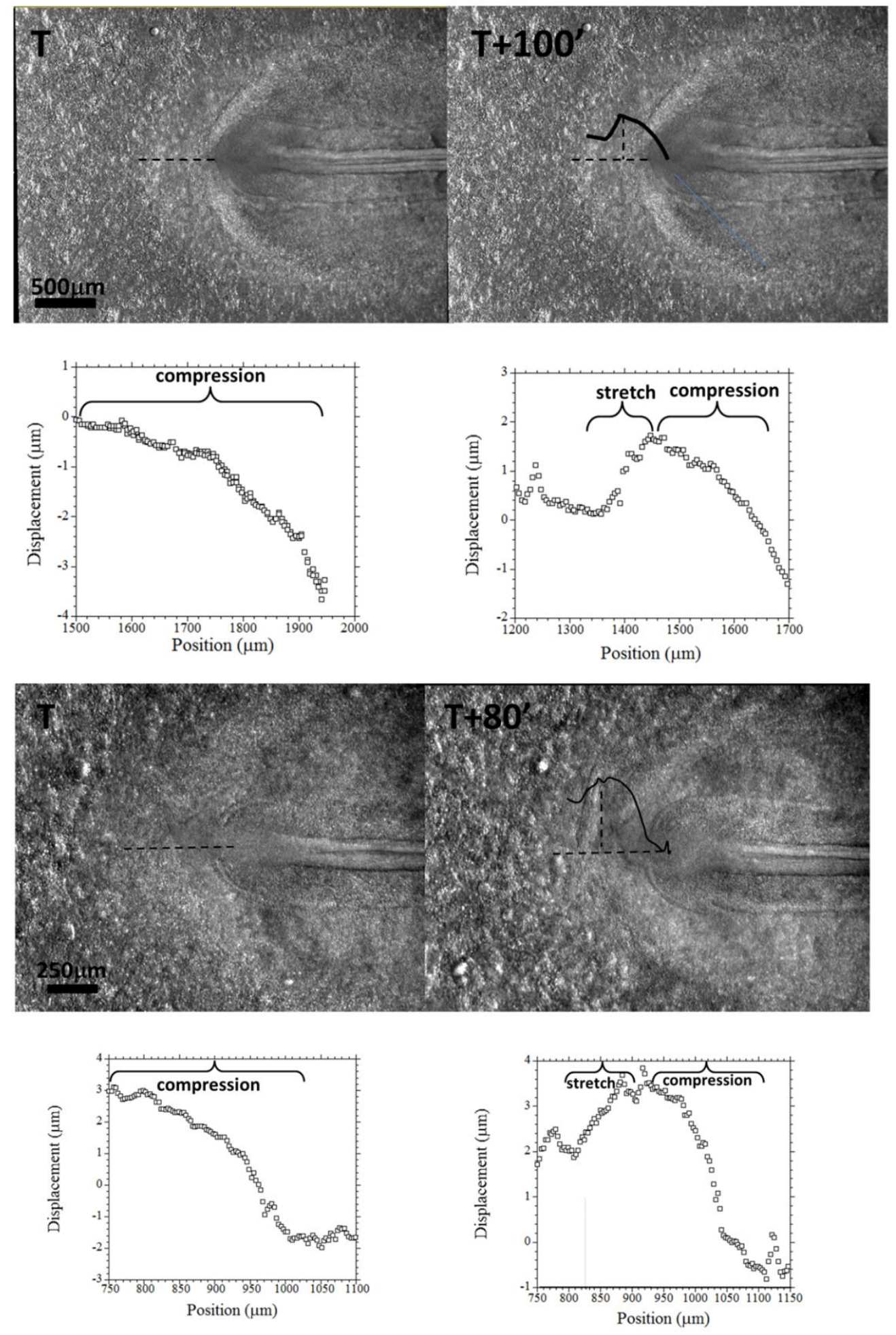

Figure 3b 
Figure 3 Title : Observation at Mag. 4X, and in time-lapse (TL), of the posterior area during transition from a 2D to a 3D form. Fig. 3a: Snapshots of the presumptive tail passing over the plane of the blastula (and eventually hanging over the anus), from Video 3. The anus is pointed by the black arrow. The presumptive body edge forms a circle located away from the tail bud. (DV: Dorso-Ventral). Fig. 3b Similar data for embryo in Videos 4 and 5. The graphs show the quantitative analysis of the displacements along the median axis in the anal area, along the dashed lines. The displacements are measured between the presumptive tail, and the extra-embryonic organs, across the anal area, at two times separated by a $100 \mathrm{Min}$. interval, in 3a and 3b, a 80 Min. interval in 3c. At early stages, the plot of the data shows a compression (the gradient of the displacement is negative). To the right, at a later stage, although the anal area exhibits a higher compression, the surrounding tissue is in a stretch state due to the constriction of the ring of cells which deforms the ectoderm ventrally towards and underneath the dorsal tissue. Hence the anal area finds itself squeezed between a constricting peripheral ring and a pushing tail bud. 

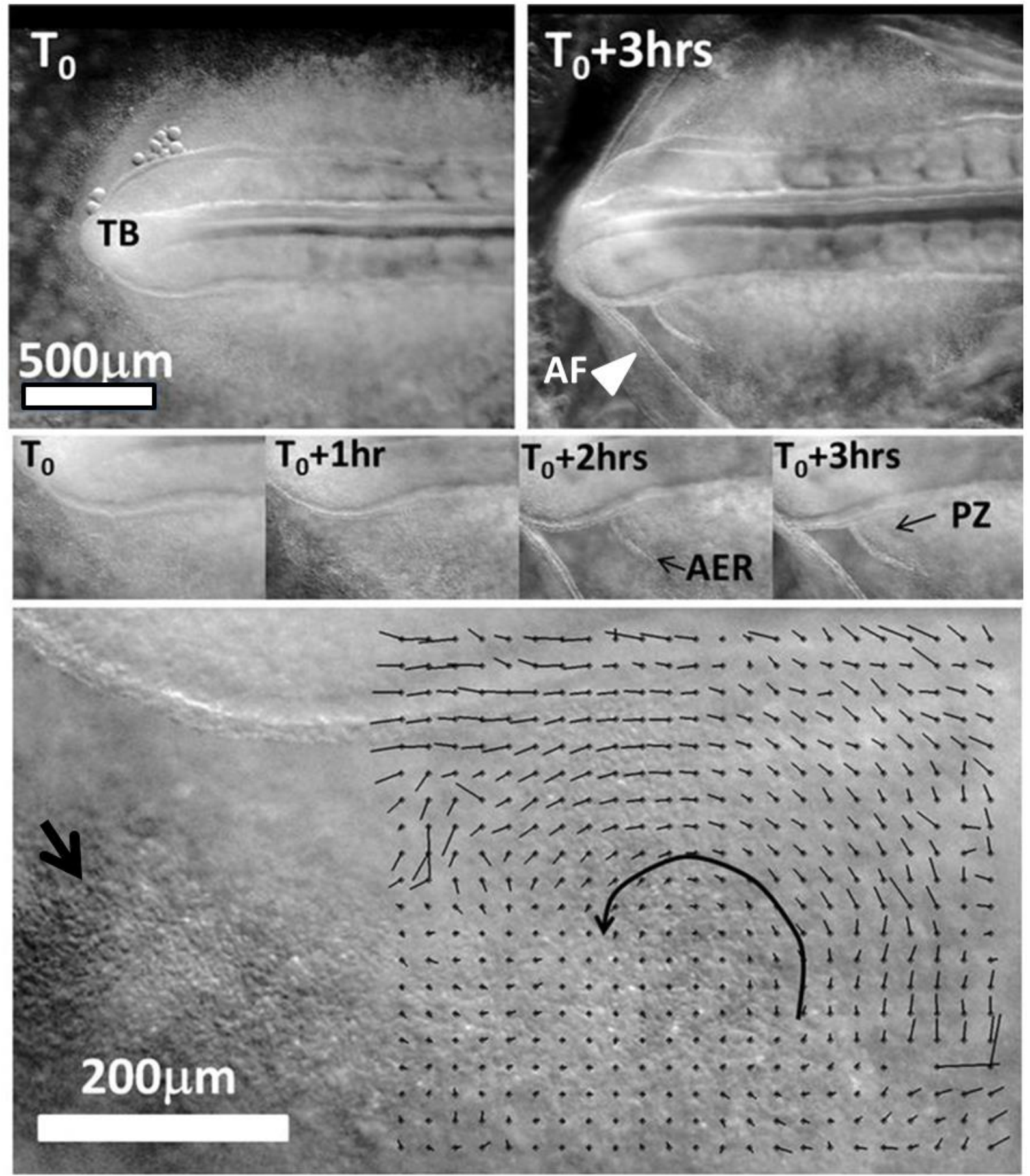

Figure 4a 


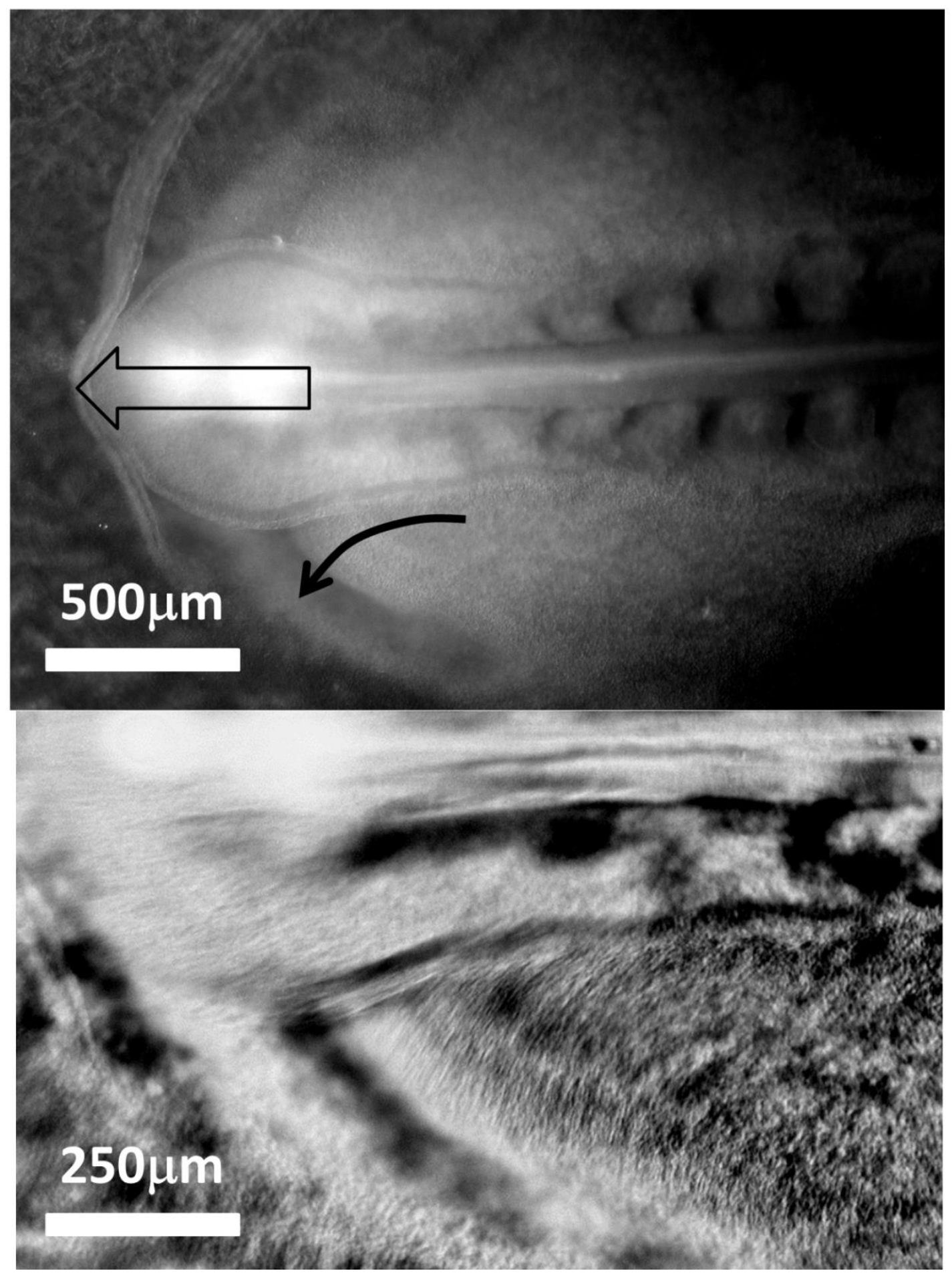

Figure 4b 


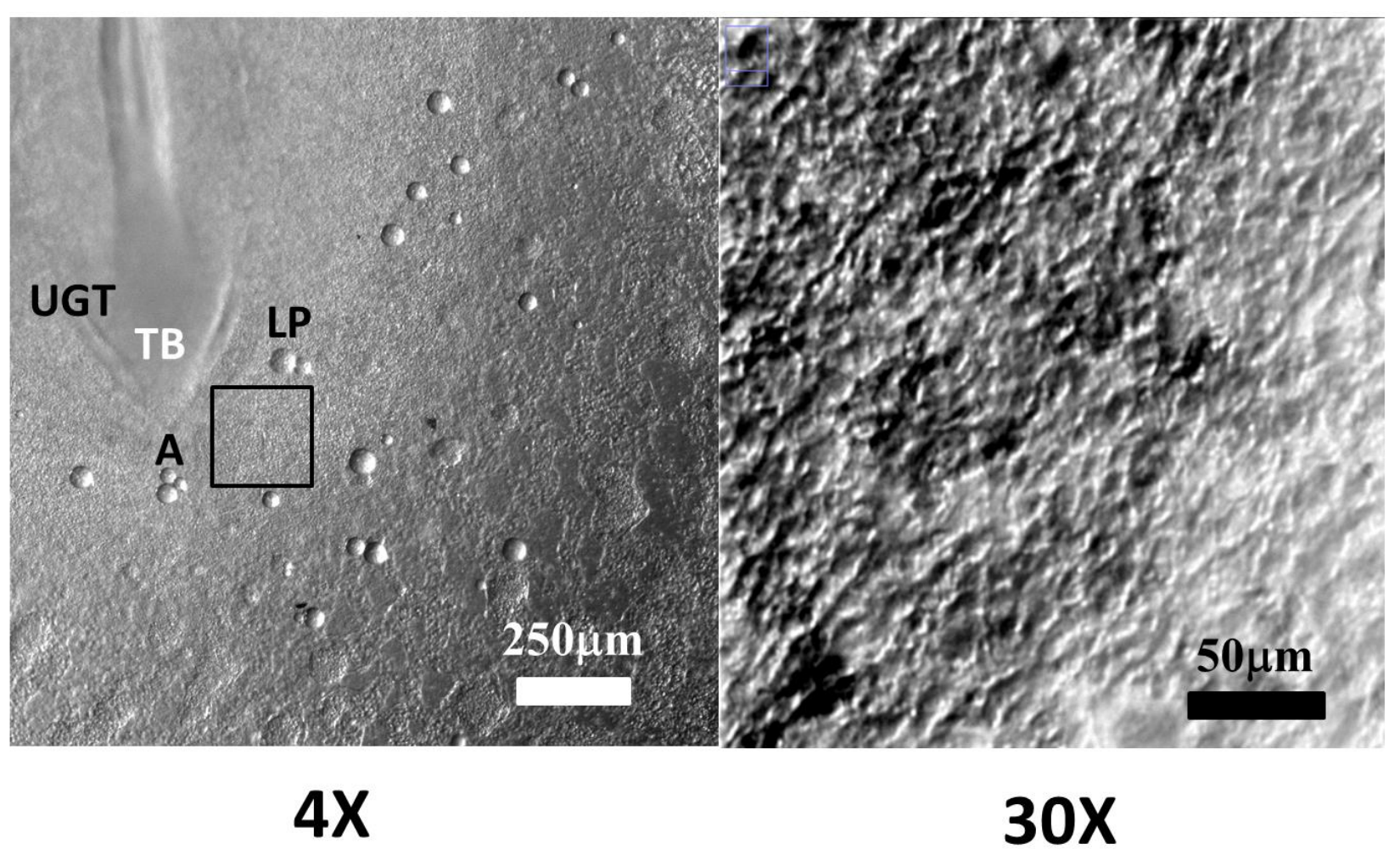

Figure 4c

Figure 4 Title : Analysis of the movements in the axilliary area of the tail bud. (From Videos 8 and 9). Fig. 4a shows 3 hours of time-lapse and the Particle Imaging Velocimetry (PIV) analysis of the posterior pull of the tail. The Video and the PIV analysis show a lateral shear of the dorsal limb ectoderm by the tail. We extract the movements during half an hour of tissue flow (the length of the movements indicated by the black segments is scaled to visible sizes). A very clear long ranged vortical movement is observed. This vortical drag collides against the surrounding ectoderm which constricts (it will form the ventral tissue after folding). The combination of the posterior shear and of the constriction of the surrounding tissue folds the limb edge, along which the Apical Ectodermal Ridge (AER) becomes visible. The Progress Zone (PZ), is also visible. The Tail Bud (TB) progressively reaches out towards the ring forming the Amniotic Fold (AF, white arrowhead). Fig. 4b shows a direct superimposition of the frames grabbed during 1 hour, in a similar embryo at the same developmental stage, showing the actual cell streamlines. Please note that while the tail is triggering the folding of the limb buds sideways, it also triggers the amniotic fold at the apex of the tail bud (Hollow arrow in $4 b$ Top). Fig. $4 \mathbf{c}$ shows the cell alignments in vivo. Cells are aligned in the belt surrounding the presumptive limb territory (see also area pointed by the 
arrow in Fig. 4a). AF=Amniotic fold; $\mathrm{TB}=$ Tail Bud; UGT=Uro-Genital Tract; $\mathrm{A}=$ Anus; LP=Limb Plate.
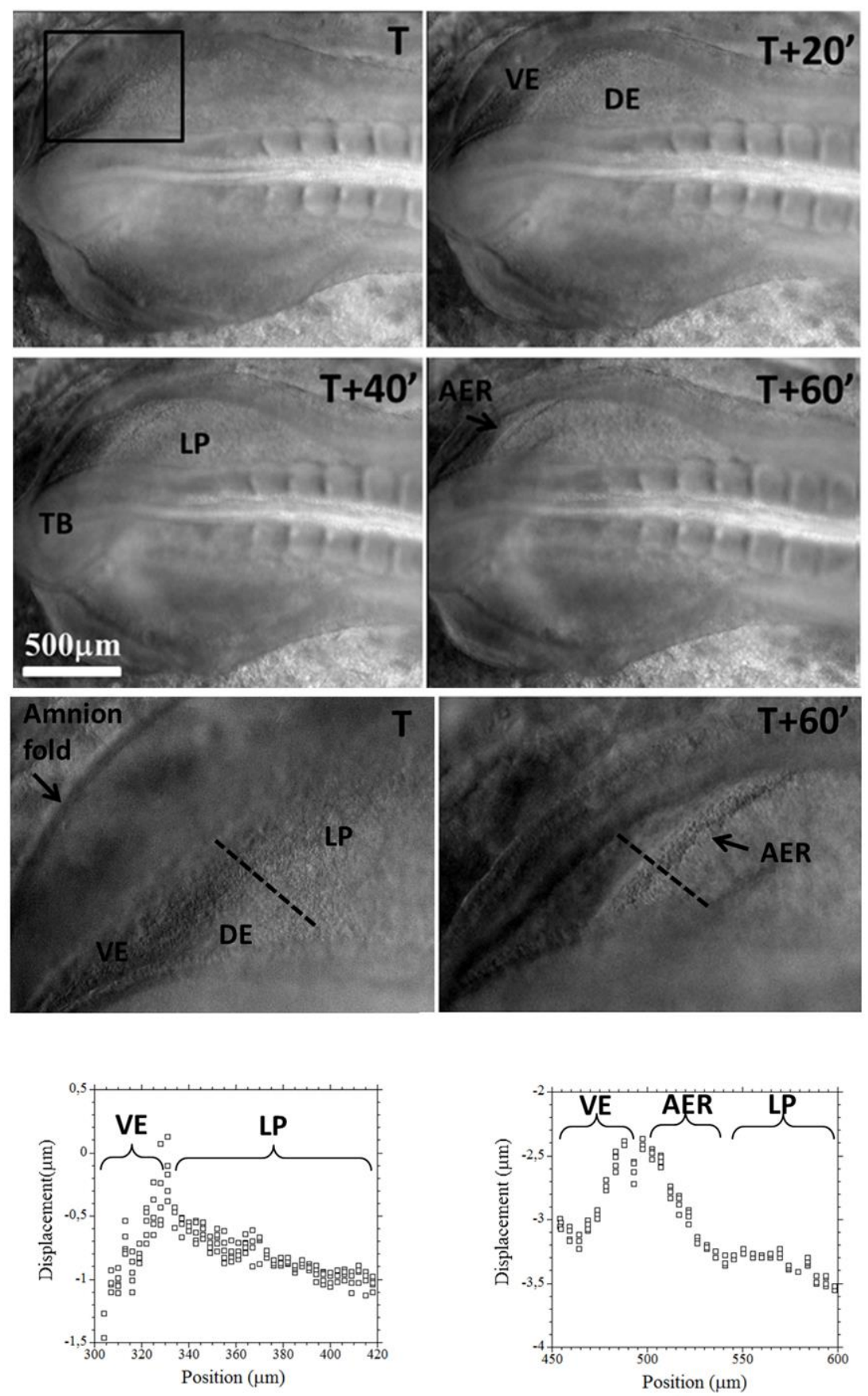

Figure 5a 

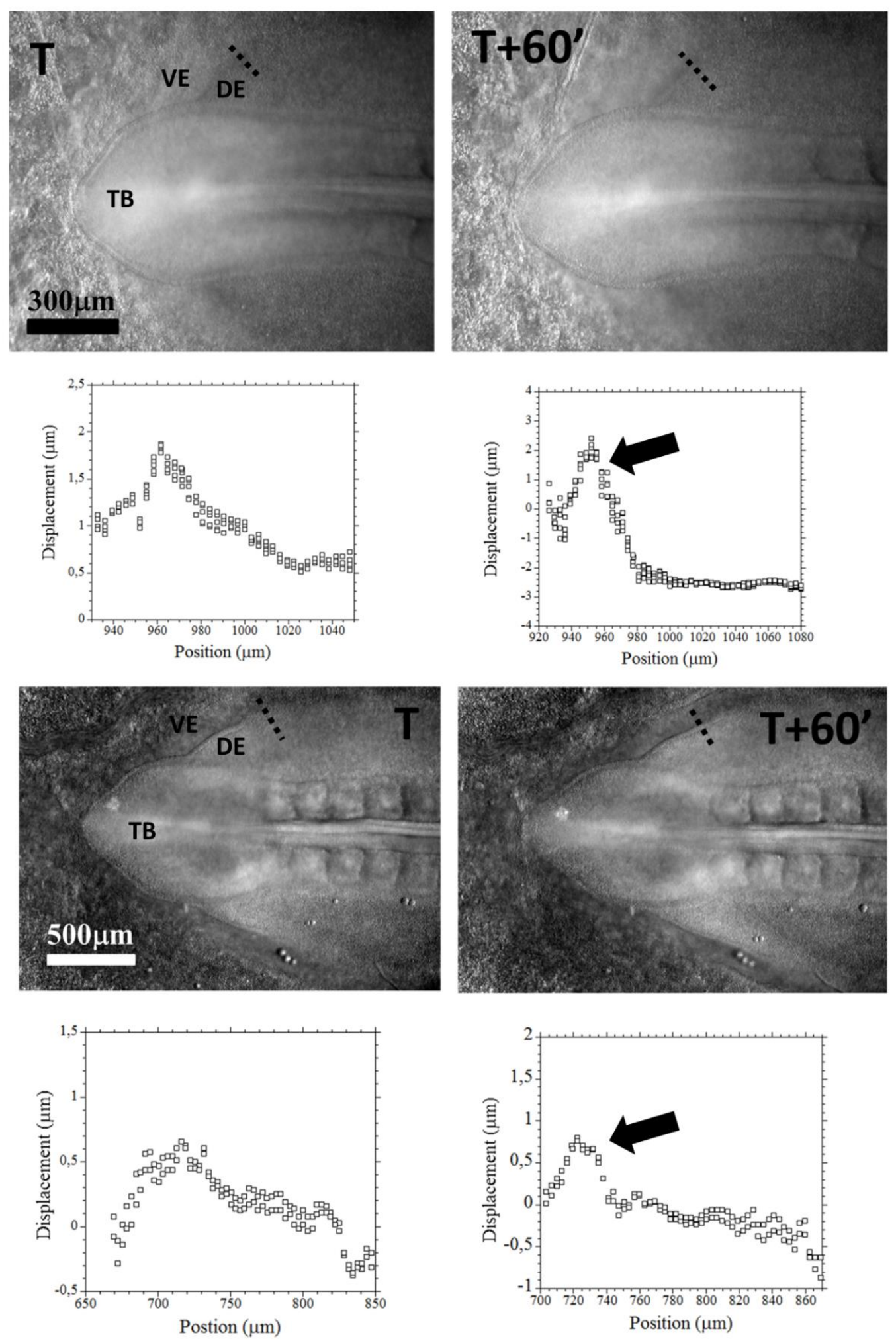

Figure 5b 


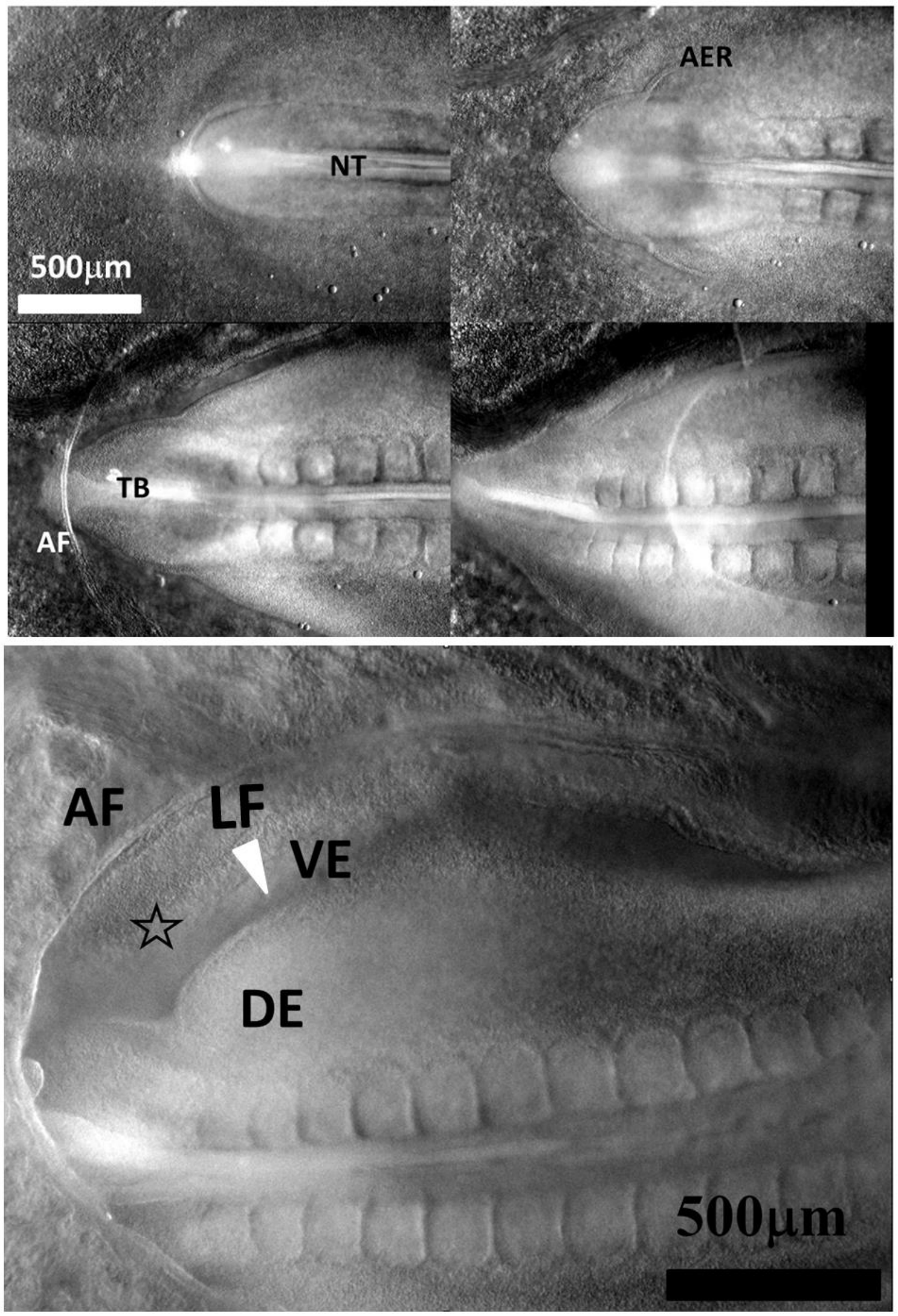

Figure 5c 
Figure 5 Title : Dynamics of limb fold formation. Fig. 5a Top : TL at Mag. 4X (from Video 10) showing the propagation of the edge of the limb bud (AER) along the boundary between the ventral and dorsal tissues. (DE: Dorsal Ectoderm; VE: Ventral Ectoderm; LP: Limb Plate; TB: Tail Bud; PZ: Progress Zone). Fig. 5a-Bottom : Magnification of the area in the rectangular frame shown in Fig. 5a-Top and analysis of the displacements perpendicularly to the edge of the limb. The ring around the VE constricts and folds the ectoderm ventrally. At an early stage, the limb territory is compressed uniformly in plane (graph to the left showing a uniform monotonic decrease of the displacements over the Limb Plate), while the surrounding tissue is stretched by the contraction of the peripheral ring. One hour later : the AER forms when the fold wave passes by the edge, and is correlated with a larger centripetal deformation along the edge of the LP (Right). Fig. 5b Two other examples showing a similar behaviour in different embryos (following the request of an anonymous reviewer, from Videos 11 and 12). Although there exists some variability in the exact dynamic pattern, the observed mechanism is the same : the contraction of the ring surrounding the presumptive edge compresses the presumptive limb bud, and a rapid localised condensation occurs along the limb edge (arrow), associated to the formation of the Apical Ectodermal Ridge. Fig. 5c Complete TL movie of the formation of the Limb Fold (LF, white arrowhead), and of the Amniotic fold (AF), from Video 12. The tail bud first passes over the average plane, while the limb tissue folds underneath, and next passes underneath the average plane while the amnion folds over the tail bud. The star indicates the area where the belts of aligned cells are found. 


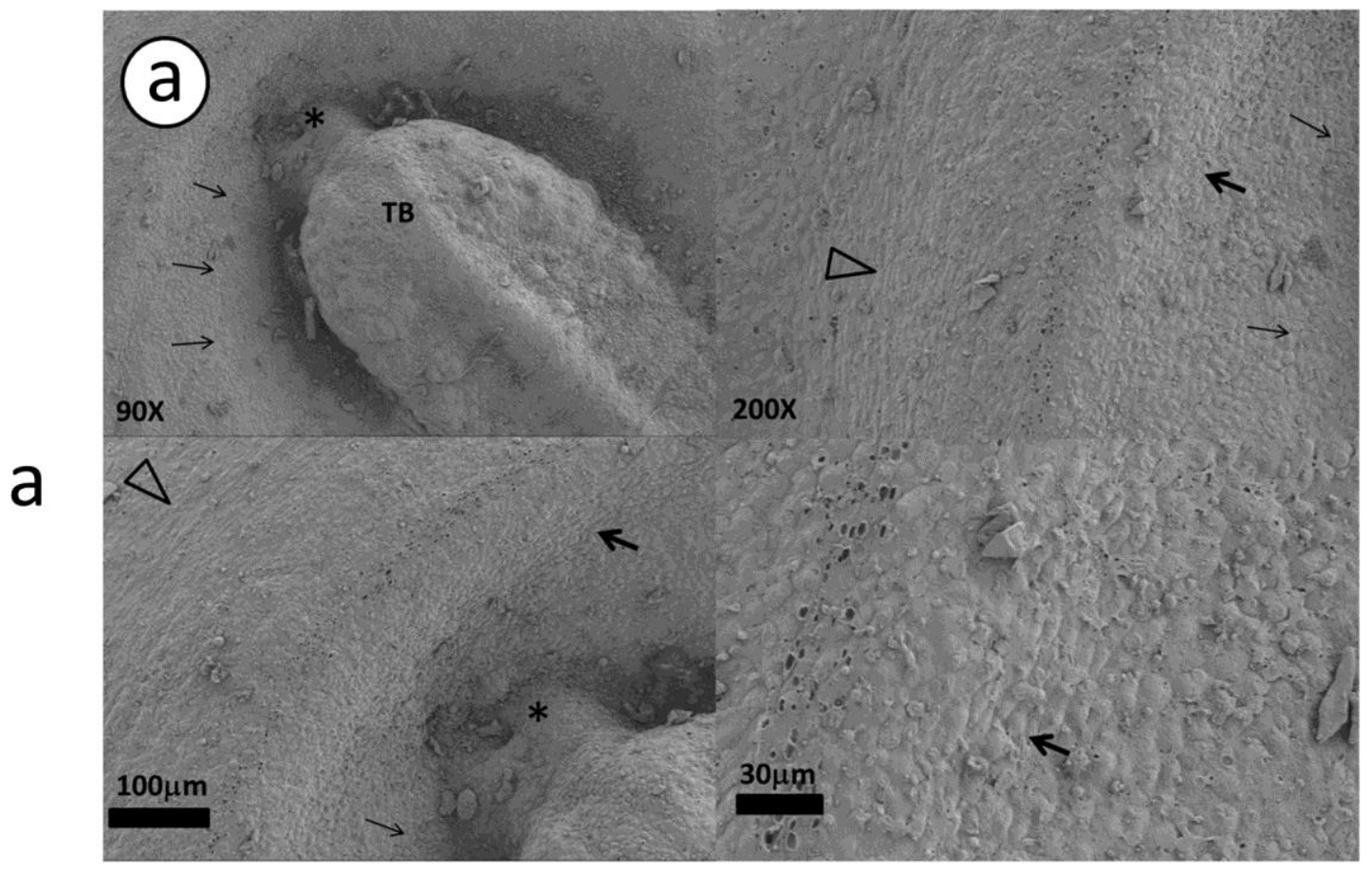

Figure 6a 


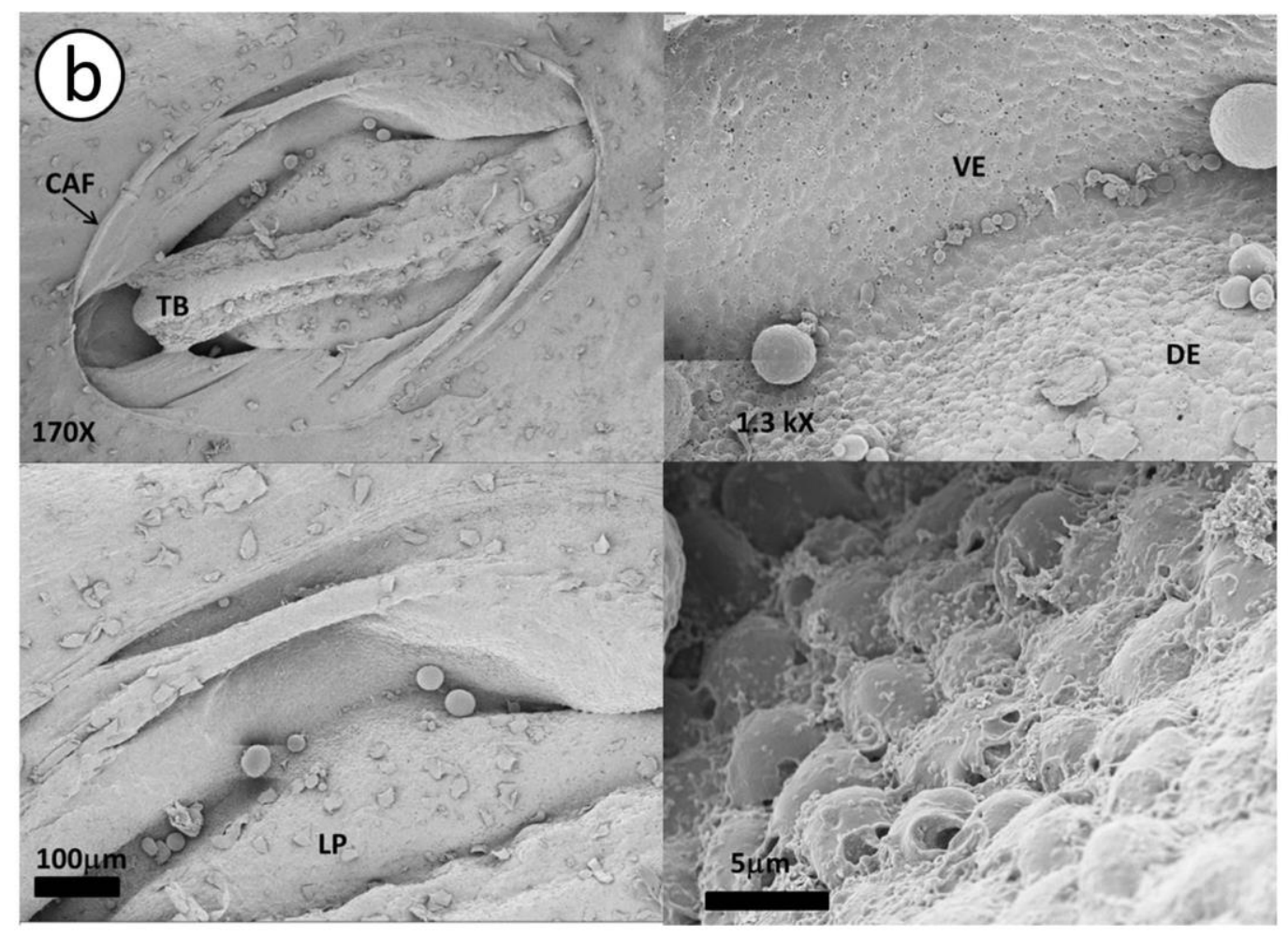

Figure 6b 


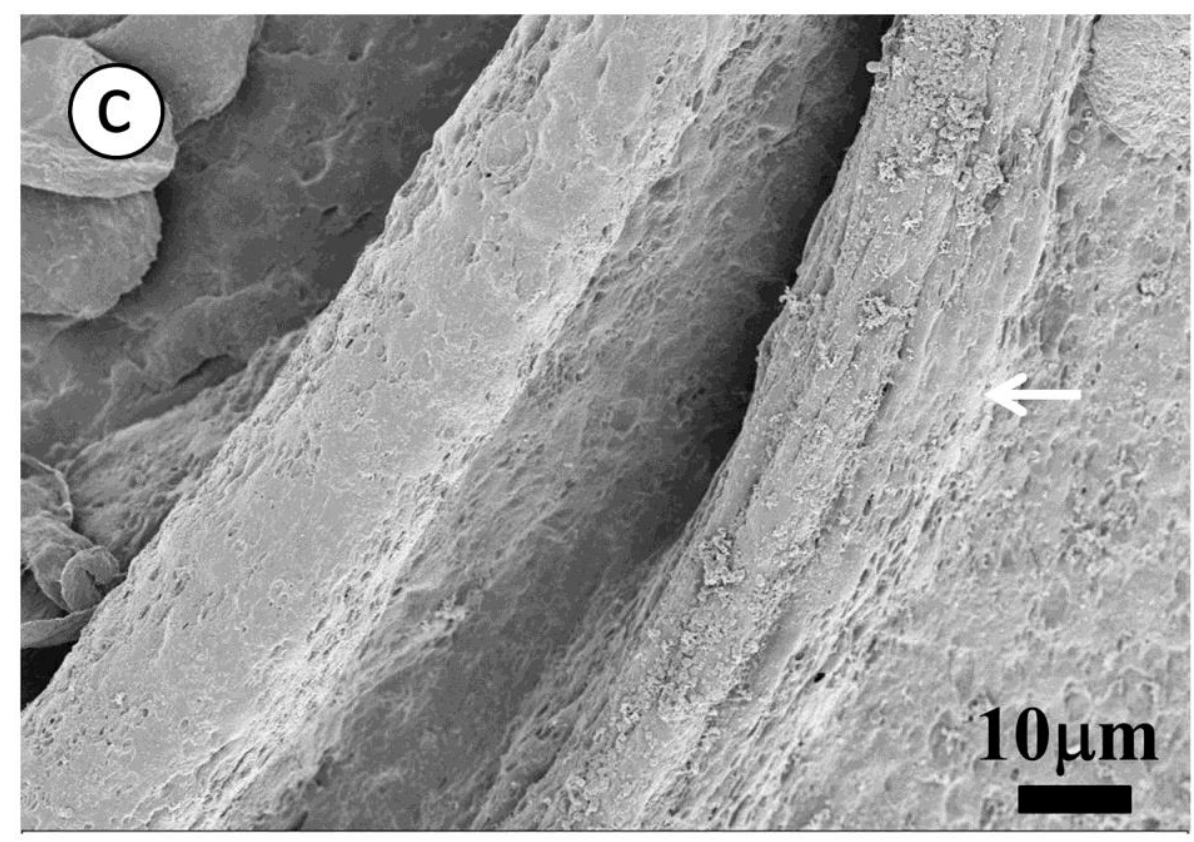

Fig. 6c

Figure 6 Title : SEM of embryos during formation of the limb fold. (LP: Limb Plate; TB : Tail Bud; DE : Dorsal Ectoderm; VE Ventral Ectoderm ; CAF Chorio-Amniotic Fold). In Fig. 6a (the tail bud is not yet "post anal"), one evidences two zones of oriented cells : there exists one belt of cells (hollow arowheads) beyond the body boundary, which will actively form the chorio-amniotic fold. More internally, a second belt of aligned cells (bold arrows) is present, which will form the dorso-ventral fold of the embryo. Careful inspection allows one to identify the boundary between the dorsal and ventral ectoderms, with cells in the DE being larger and more contrasted (thin arrows) than in the VE. In Fig. 6b one sees the embryo during folding of the dorso/ventral boundary (the tail bud hangs "post-anally"). Care was taken to fix an embryo rapidly, exactly at this crucial moment. The fold is partially formed, and one evidences that the fold is advanced in the posterior area, and in the anterior part of the limb territory, while the folding process is less advanced in the middle. A zoom in that area allows one to see both Dorsal and Ventral ectoderms, to image the obvious difference in cell sizes, fiber content, and also that the fold is starting to form in that area, exactly over the boundary between the two cell territories. Fig. 6c shows a close up view of supra-cellular alignments and folds. 

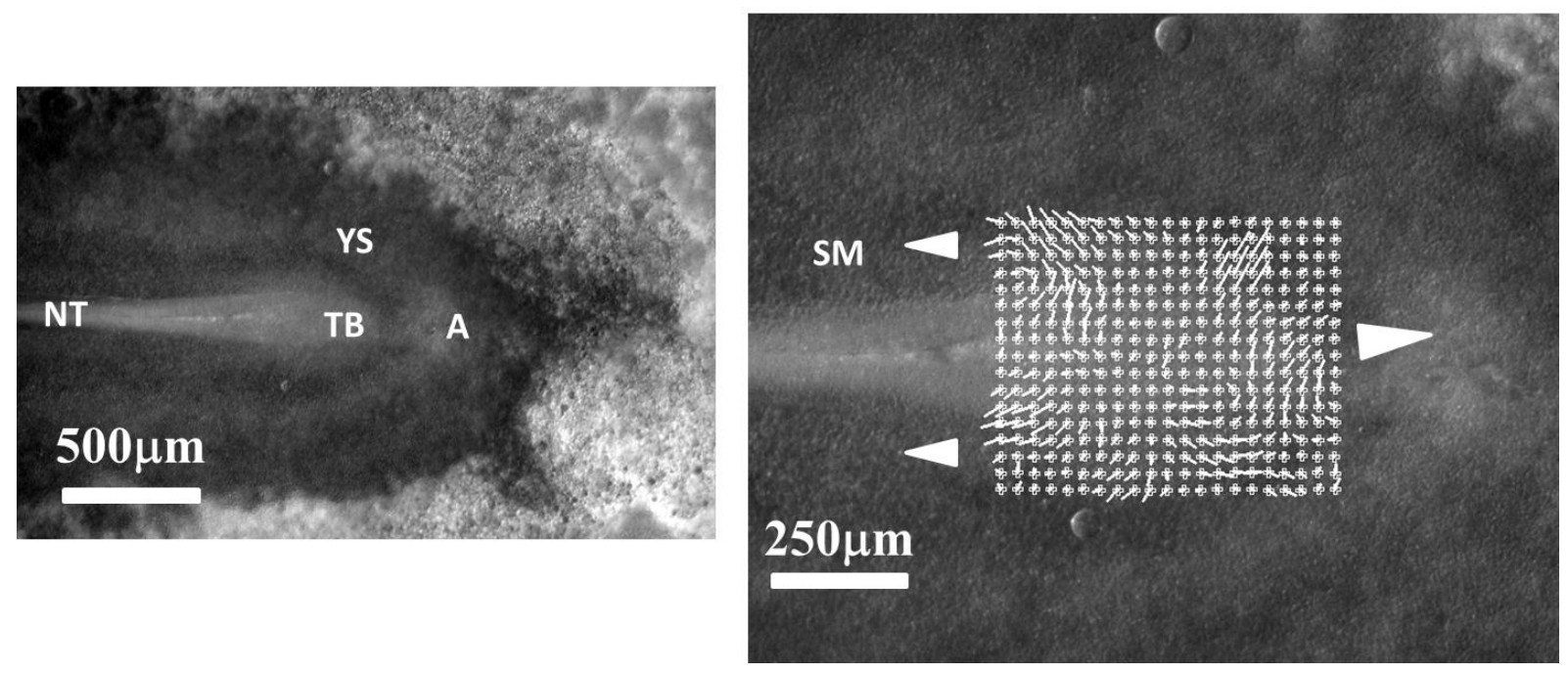

Figure 7a

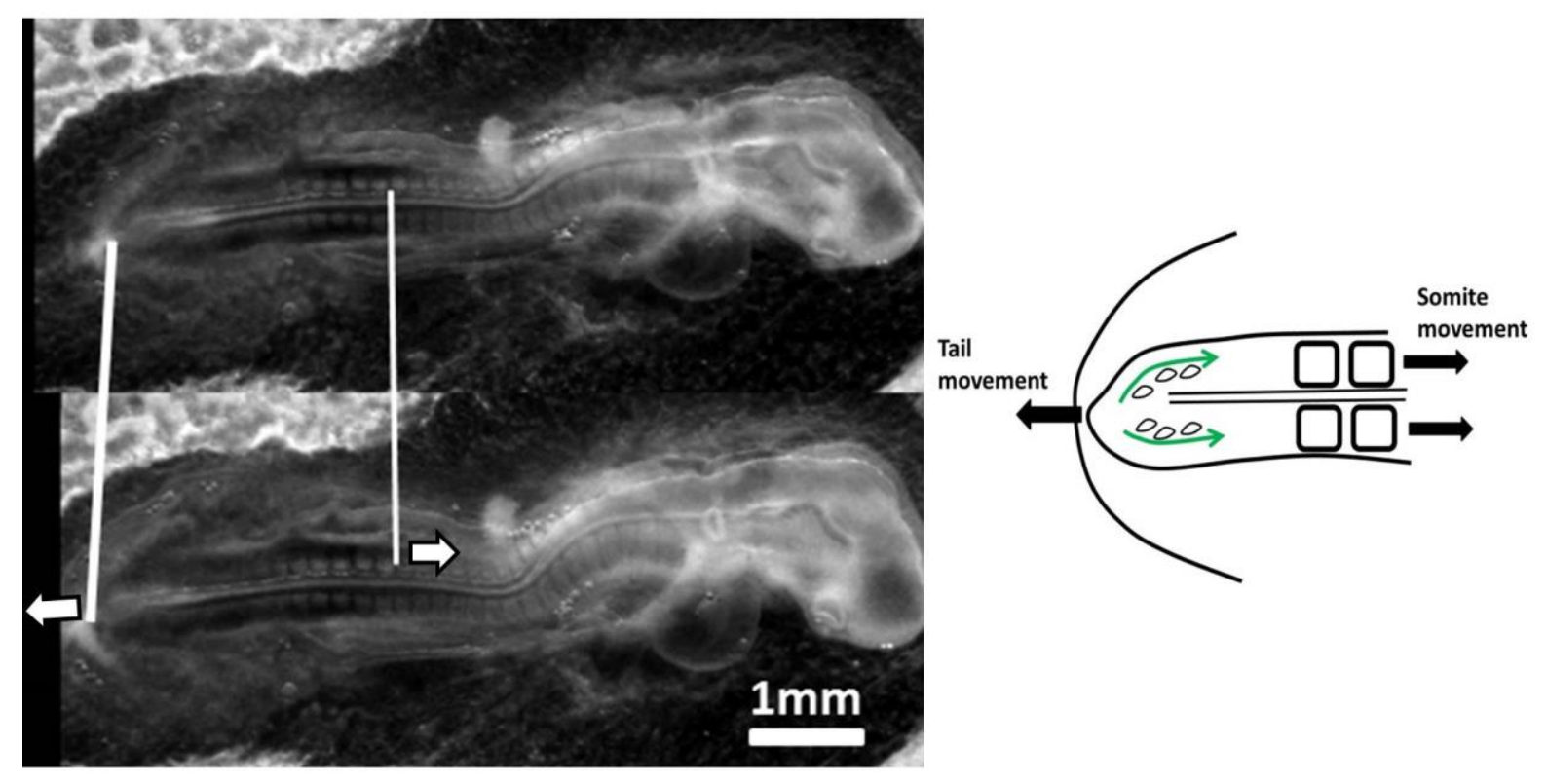

Figure 7b 

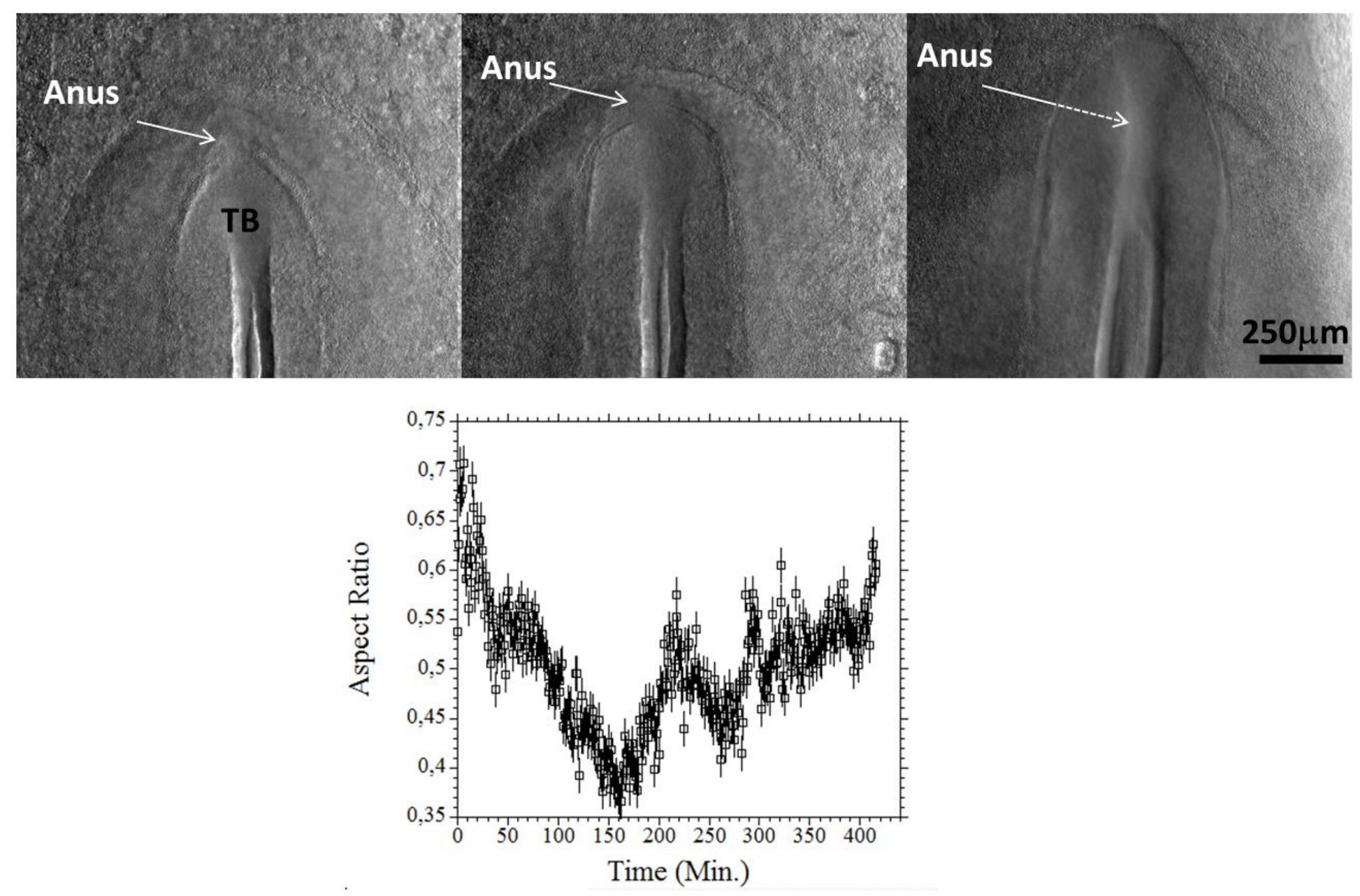

Figure 7c

\section{Figures 7 Title : Dynamics of tail extension during limb edge folding.}

Fig.7a Cellular movements during tail extension. The plate shows the PIV analysis of cell movements during 5 minutes of Time-Lapse, during formation and posterior movement of the presumptive Tail Bud (the dimension of the displacements have been scaled to visible sizes). As the presumptive tail bud extends posteriorly, there actually is a cell flow oriented anteriorly. (YS=Yolk Sac, NT=Neural Tube, TB=Tail Bud, A=Anus, SM=Somitic mesoderm). Fig.7b Two snapshots at low resolution of a developping embryo, showing the movement of the presumptive vertebrate precursors. The somitic tissue (or "somitic mesoderm") is advected anteriorly at long range during embryo development, while the tail extends (see Video 13). Fig.7c-Top Snapshots showing the tail form, during the pre-anal to post-anal transition. The apex of the tail is first "sharp", when it s far from the anus ; then "flat" as it pushes against the anus ; then "sharp" again after passing over the anus . Fig.7cBottom : Following the aspect ratio of the tail bud (ratio Height/Width, measured manually at the same distance from the tip) shows a steady flatenning, during the pre-anal phase of 
compression, and next, a sharpening in the post-anal phase, with a very rapid transition, and actual oscillations. We ascribe this effect to elastic compression of the tail against the anal region, followed by a brutal fold and the release of the elastic energy after passing over the anal pit (the same phenomenon is also quite visible in Video 5). 

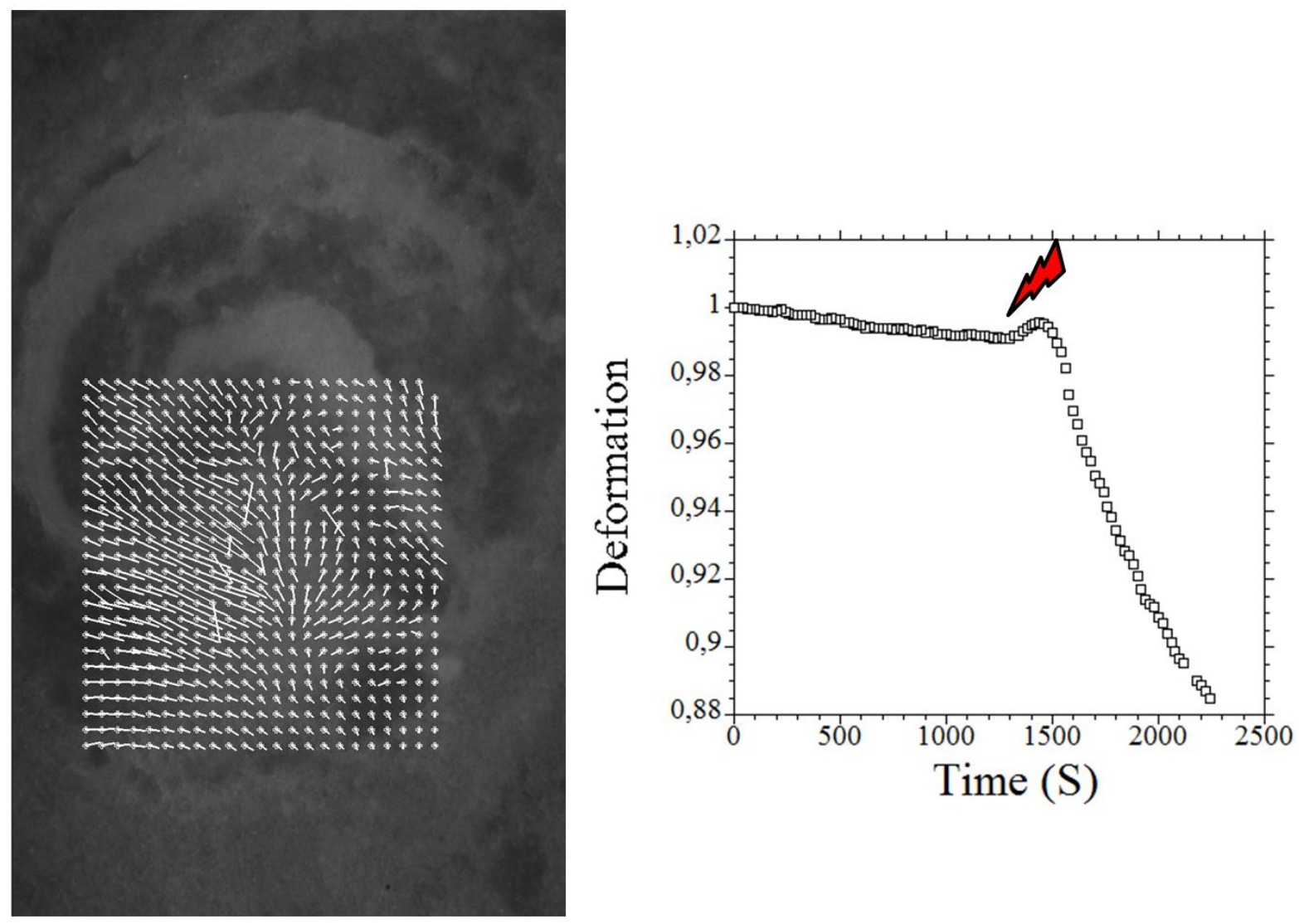

Figure 8a
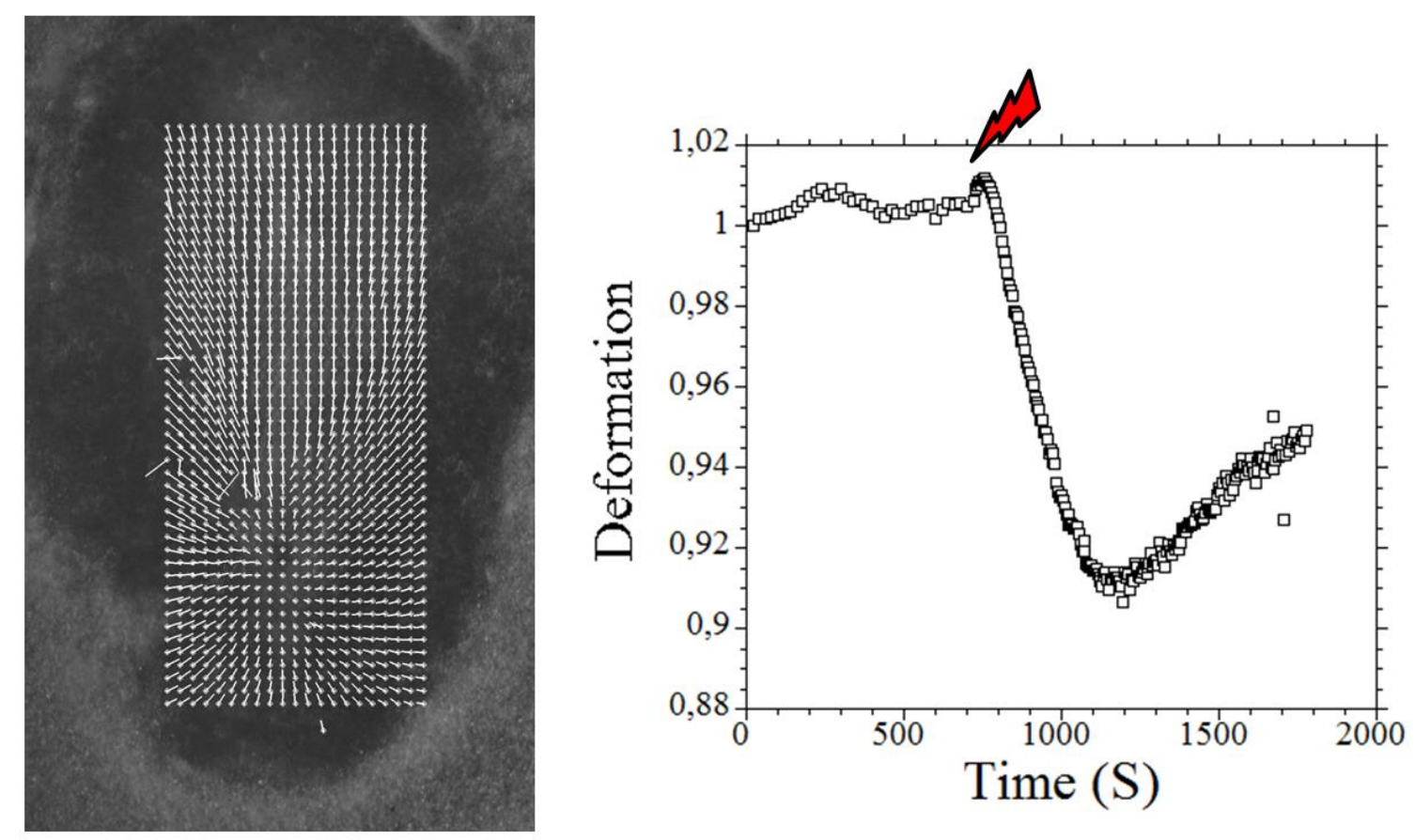

Figure 8b 
Figure 8 Title : Electric shock at the blastodisc, and early neurulation stages. Short electric shocks $(8 \mathrm{Sec}$. at $1 \mathrm{~Hz})$ of amplitude $1.5 \mathrm{~V}$ were applied with a thin $(50 \mu \mathrm{m})$ positive copper electrode. The "lightning" symbol shows when the electric stimulation was applied. Fig. 8a : The map of tissue movement at early gastrulation stage, just after the electric shock, in the area where the electric shock was applied. To the right, the deformation (in the contracting direction, close to the stimulation point), as a function of time, showing an increase in deformation rate of order 20X (see Video 14). Fig. 8b : The map of tissue movement at early neurulation stage, just after the electric shock, in the area where the electric shock was applied. To the right, the deformation (in the contracting direction) as a function of time (see Video 15). 

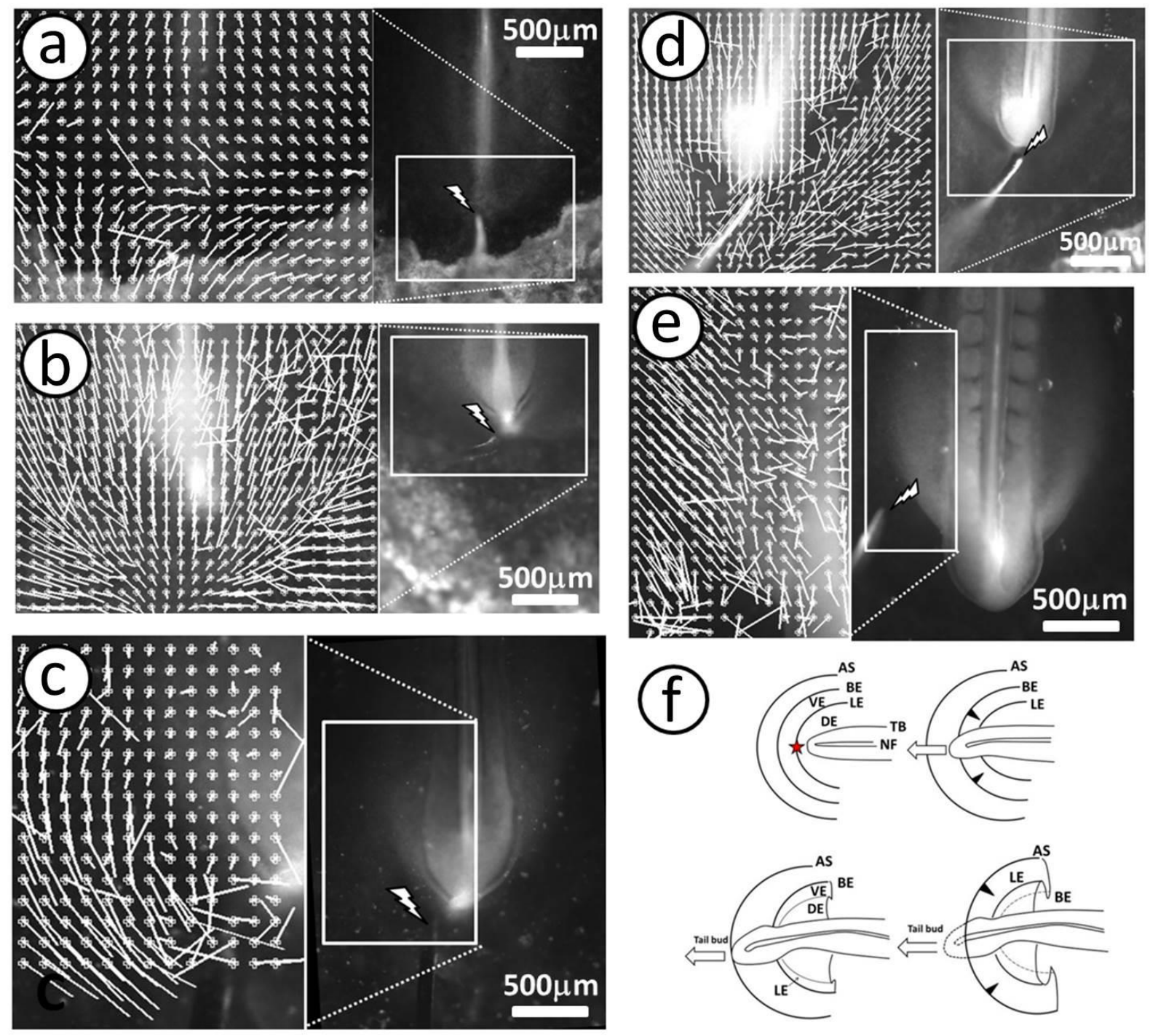

(f)
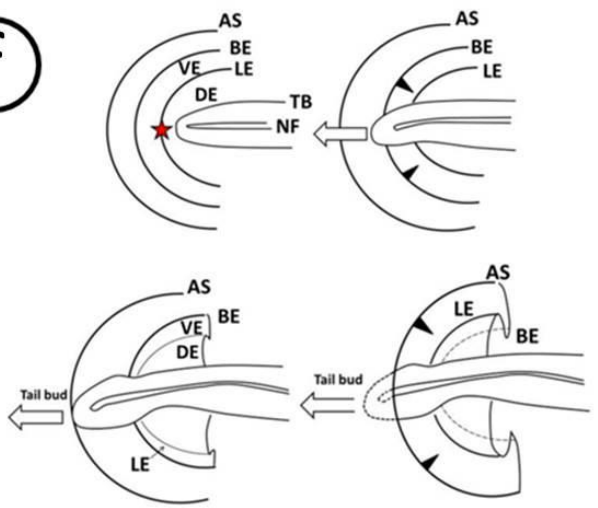

Figure 9

Figure 9 Title : Analysis of the movements following electrical stimulation around limb formation. Prior to limb edge folding, the stimulation was applied in the anal area. (a-d). 
While the limb edge was folding, the stimulation was applied laterally over the belt of contracting cells (e). In all cases, a rapid contraction was obtained which accelerated the wrapping of the tissue ventrally, and the formation of the limb edge (see Videos 16-20). Also, the amnion fold would form by a few minutes following an electric stimulation, instead of forming the next day. The plates show the massive displacement obtained around the presumptive limb edge by the electric stimulation, as extracted by Particle Imaging Velocimetry. The scheme in the bottom right shows the principle of formation of the limb edge. Initially, the blastula has a structure of encased rings. At the moment of formation of the limb bud, the central ring has already formed the neural fold (NF). This neural fold extends posteriorly, facing a succession of encased rings, which form the presumptive Dorsal Ectoderm (DE), Ventral Ectoderm (VE), and Amniotic Sac (AS). The stimulation of these rings by the tail bud in the anal area (star) provokes first the ventral fold of the body by a centripetal movement symbolized by the black arrowheads, and next the dorsal fold of the amnion by an analogous centripetal movement. The DE and the VE fold along their common boundary, while the body edge closes ventrally. After passing over the anal and limb territories, the tail bud continues to grow underneath the amnion fold, which forms a sac, as it closes up over the embryo.
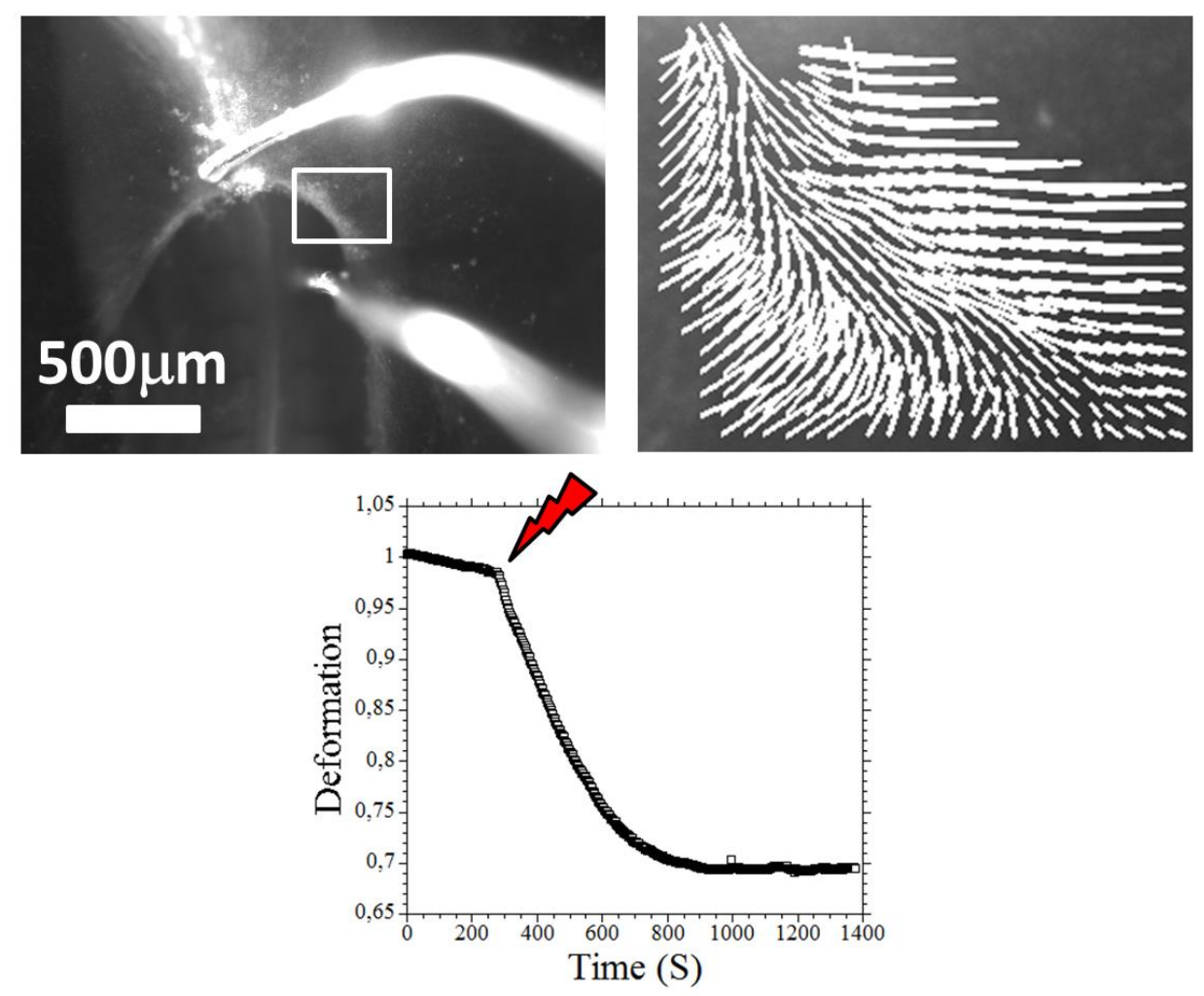
Figure 10a
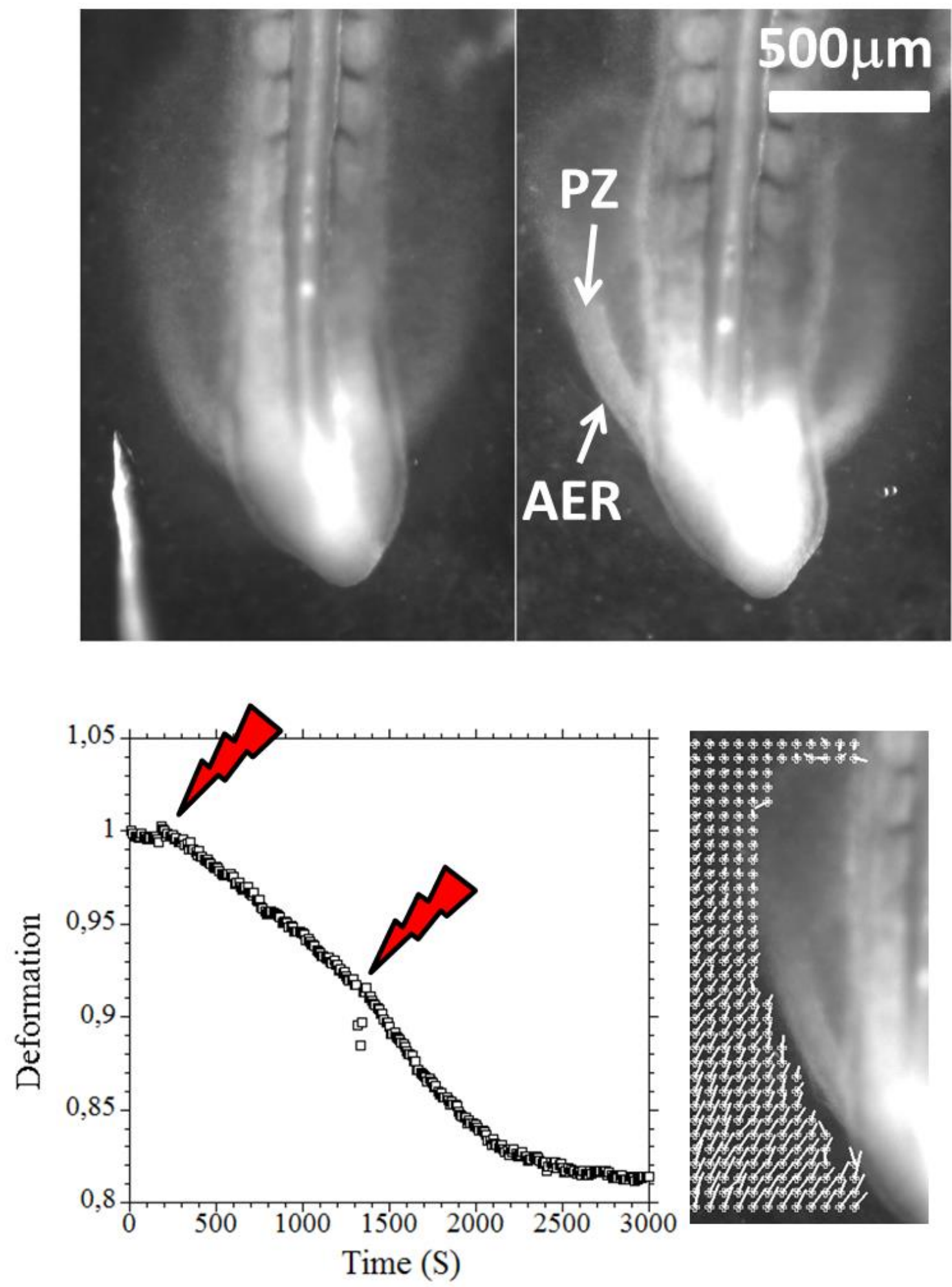

Figure 10b 

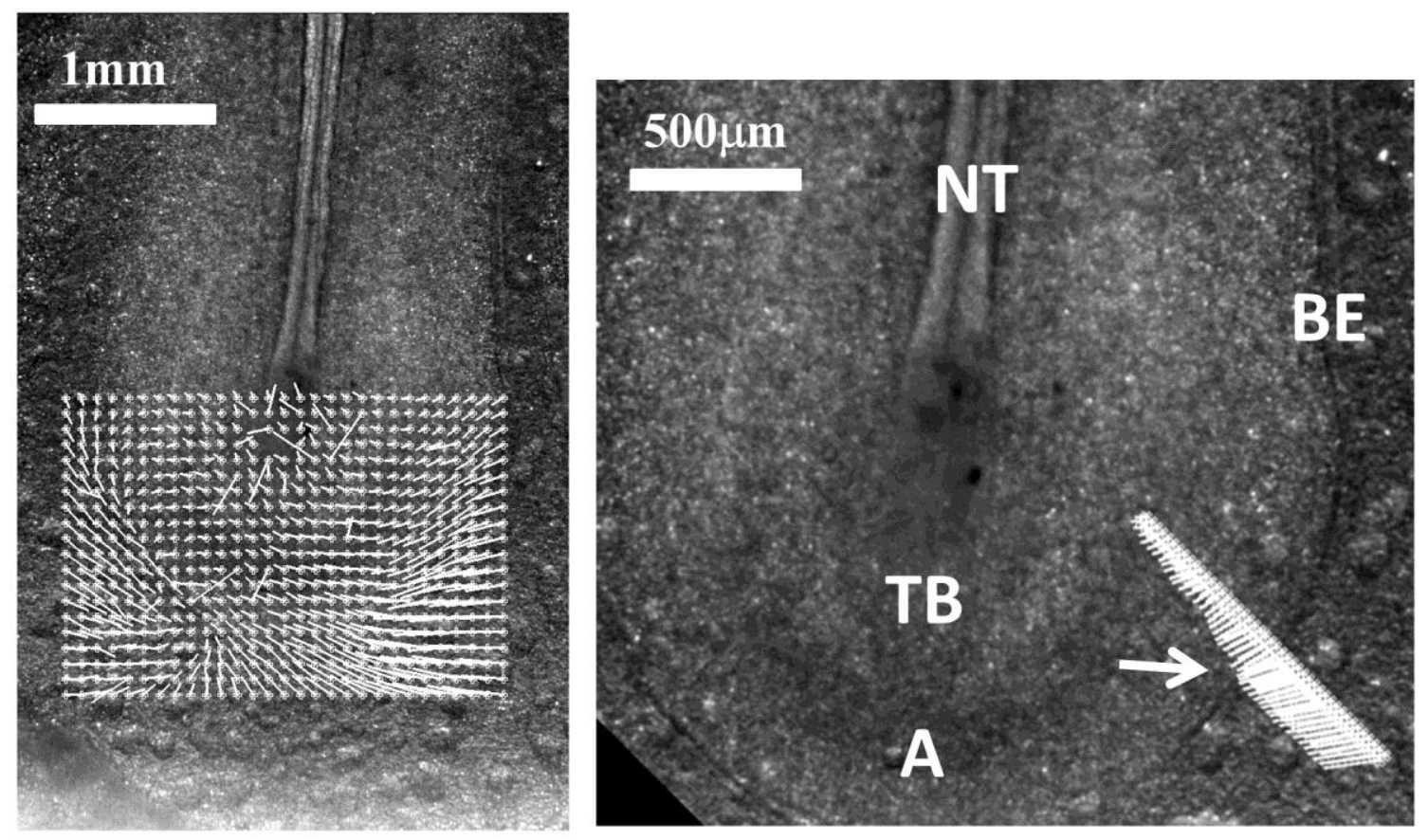

Figure 10c

Figure 10. Title : Quantitative measurement of force magnitude increase during electric stimulation. We record the deformation of the tissue prior to an electric shock and following the electric shock (the deformation is defined as the distance between two points aligned in the direction of main visible contraction). Fig. 10a shows the change in deformation rate for a shock on the amniotic sac (from Video 21), an increase by $9 \mathrm{X}$ of the deformation rate is obsreved. Fig. 10b shows the change in deformation rate for two subsequent shocks close to the presumptive limb edge (from Video 22). The moment of the shock is symbolized by a "lightning" symbol. One clearly observes an instantaneous increase of the deformation rate (slope of the data) by a factor of order $\sim 5$ and $\sim 8$. Along the presumptive limb, the electric stimulation accelerates the formation of the limb edge, the appearance of the AER and of the Progress Zone. Fig. 10c shows an more detailed analysis of the magnitude of the stimulated movement (from Video 23). Left, a wide view of the contraction pattern, Right, a refined PIV analysis along a line crossing the presumptive limb territory, and the presumptive body edge. A visible discontinuity of the movement is visible (arrow) exactly at the boundary between the presumptive limbs territory, and the body edge. The amplitude of the movement is greater 
along the belt surrounding the limb territory. (NT=Neural Tube; $A=A n u s ; T B=T a i l$ Bud; $\mathrm{BE}=$ Body Edge) 


\section{Supplementary Material (Figures)}
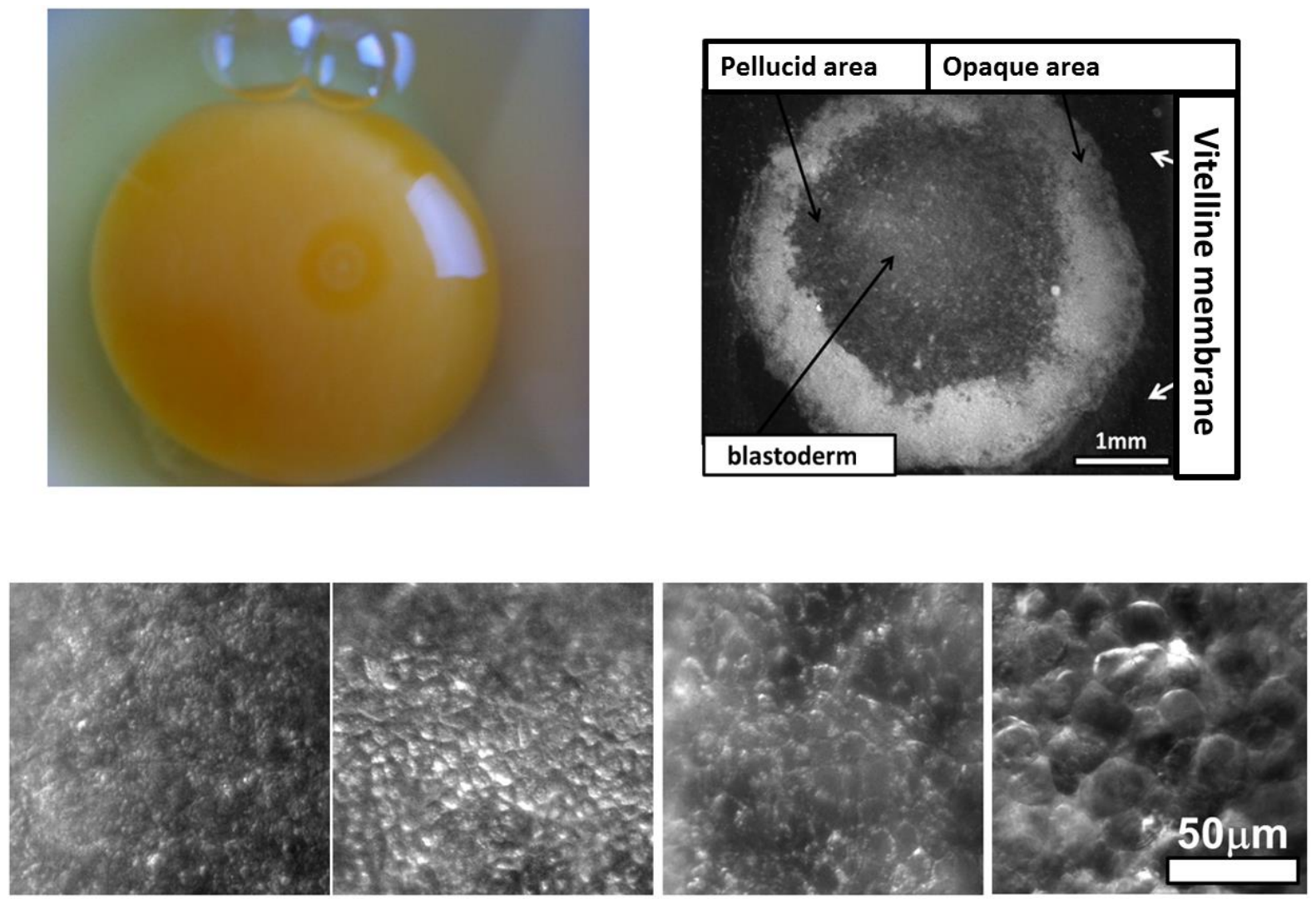

Supplementary Material Figure S1 Title : Structure of the "day 1" chicken blastula.

Reprinted and adapted from Refs. 22 and 23. Top left, an egg showing a day-one blastula (or chicken "blastodisc"). Top right, such a blastula, as dissected off the egg, rinsed, and placed in a Petri dish. One readily sees the ring structure, the Opaque Area and the Pellucid Area. The embryo body forms from the central disk called Blastoderm. Bottom: the ring structure in the early embryo is associated to a sequence of cell sizes, corresponding to different presumptive territories. Central cells are small, and cells increase in size stepwise, from one ring to the next, in a centrifugal order. The central cells form the central nervous system, the next rings form the body (dorsal and ventral ectoderms), and the outer rings form the extraembryonic organs and the digestive tract. 


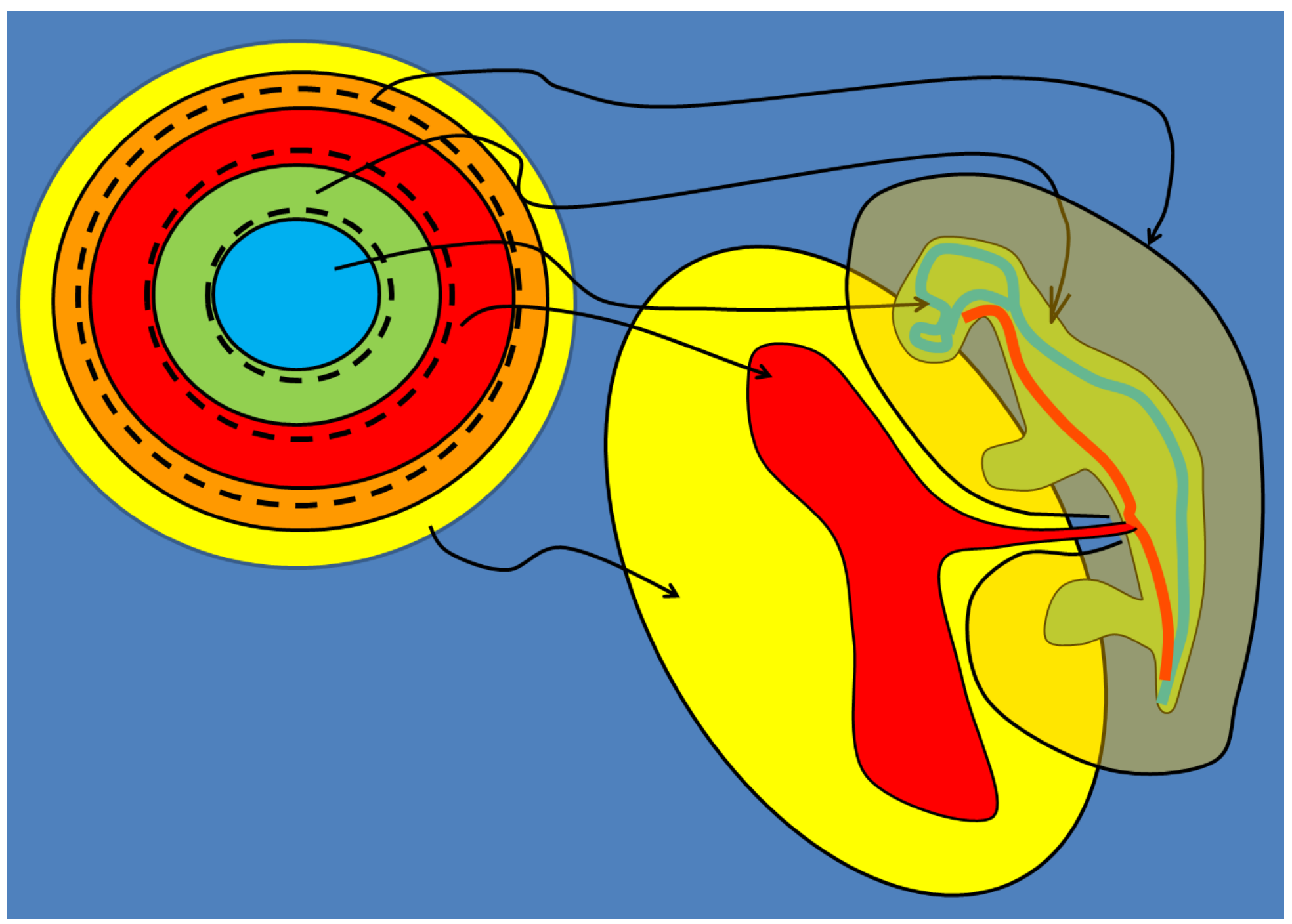

Supplementary Material Figure S2 Title : Mechanism of vertebrate formation (chicken model). Rings of cells deform and fold the blastula up to the point that it forms a recognizable animal. Each ring corresponds to a specific embryonic territory. The dashed lines represent schematically the pulling "belts" of cells. The animal forms by a cascade of folds occuring at the limits between two rings. 


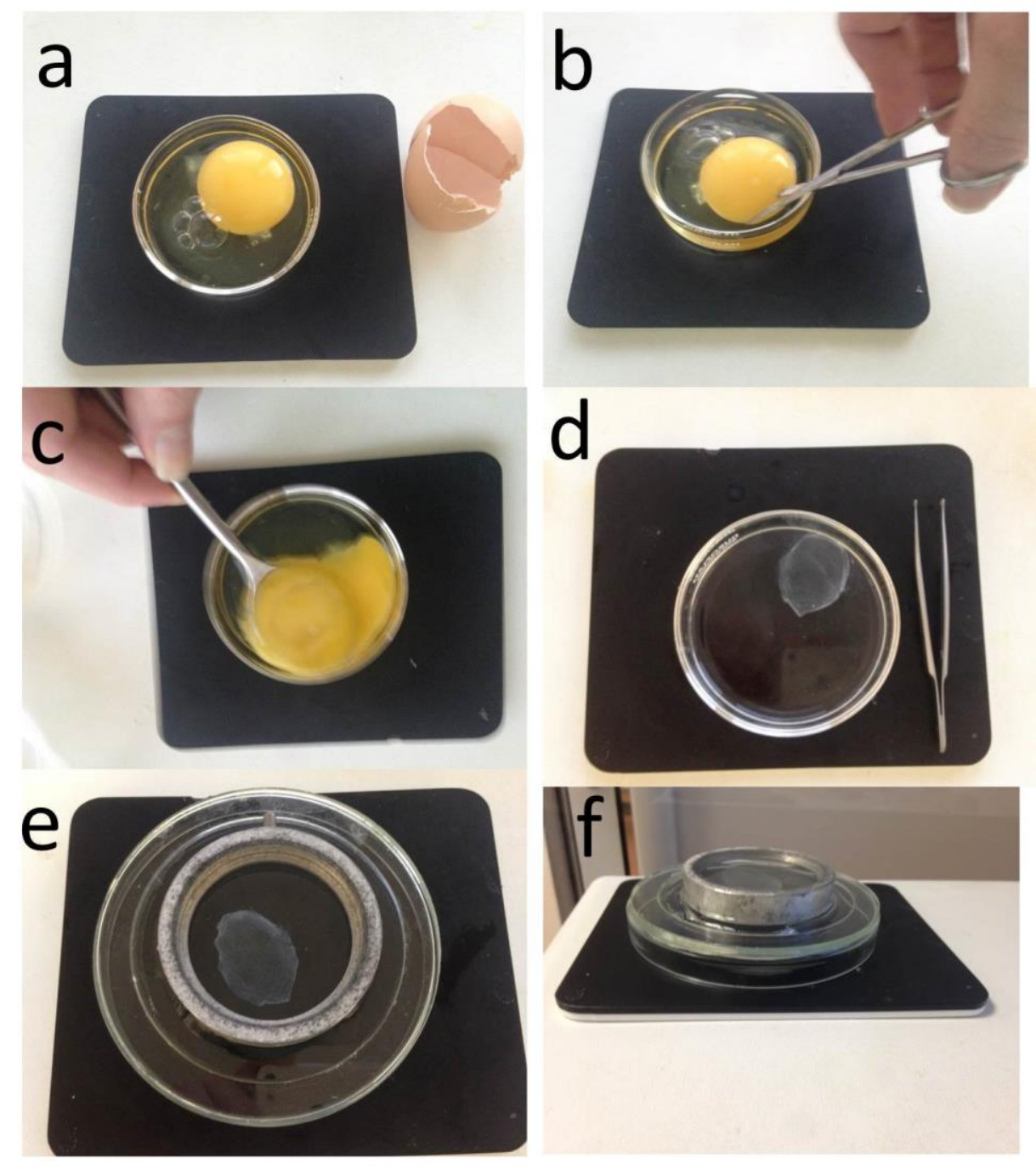

Supplementary Material Figure S3 Title : Preparation of a substrate for embryo development. A fresh egg is cracked and opened (a,b). The vitelline membrane is cut circularly (b), and the disk of vitelline membrane with its thick albumine atop is transferred to a Petri dish where it is carefully rinsed $(c, d)$. Then it is deposited upside down in a provisory bath of buffer (PBS from Dubbelco), positionned on the incubating chamber (e,f) 

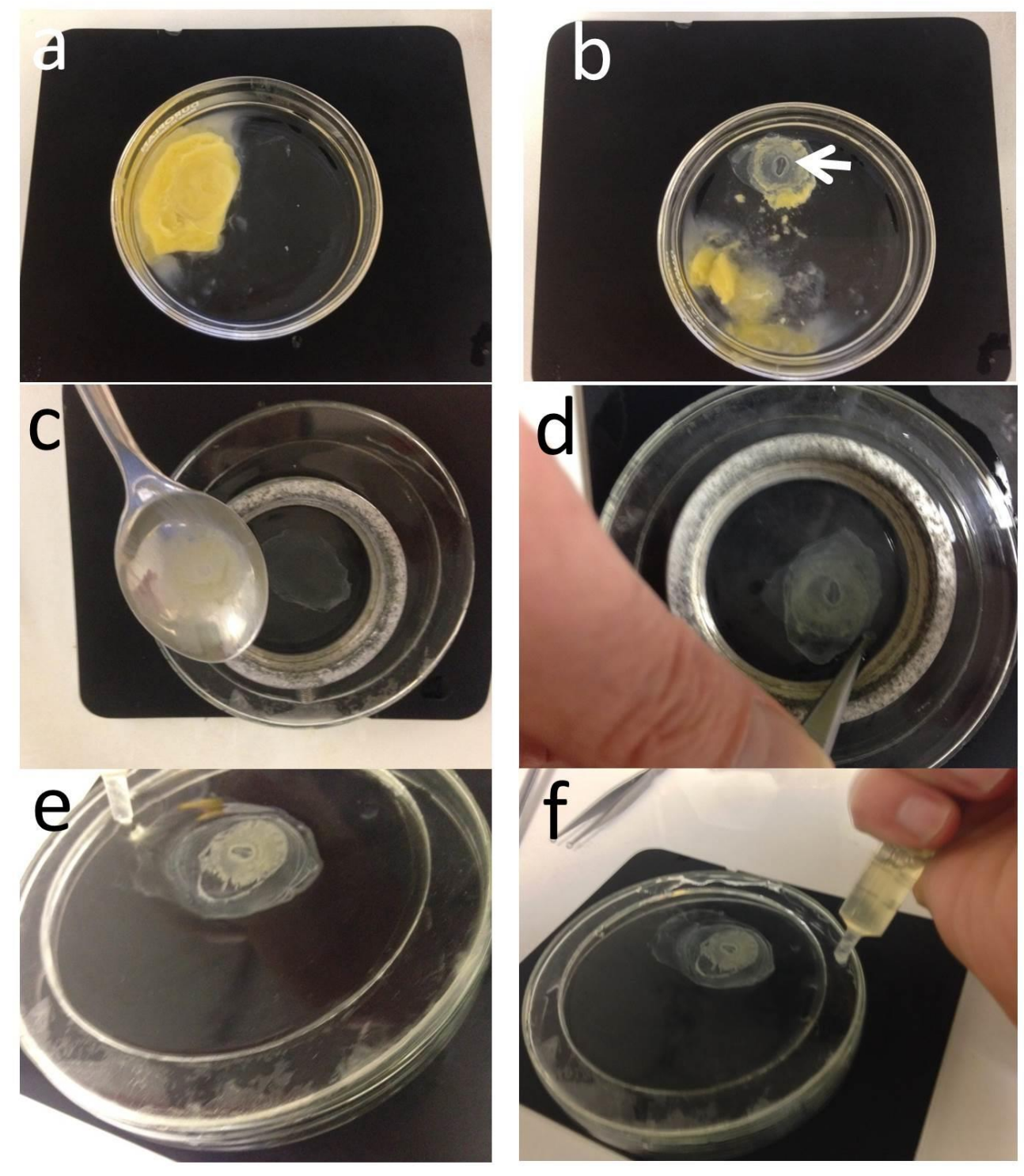

\section{Supplementary Material Figure S4 Title : Preparation of an embryo for time-lapse} imaging. An egg at proper incubation time is cracked and opened (a). The vitelline membrane is cut circularly around the embryo, and the disk of vitelline membrane with the embryo is transferred to a Petri dish where it is carefully rinsed. Then the embryo is detached from the vitelline membrane and transferred with a spoon to the provisory bath of PBS with the substrate ready (c). The embryo is laid on the substrate ad the PBS is removed while holding the embryo in place with tweezers (d). The provisory alumnum ring is removed, and albumin is deposited around the embryo (e). Albumin is also deposited along the edges of the incubating chamber, to serve as sealant. 


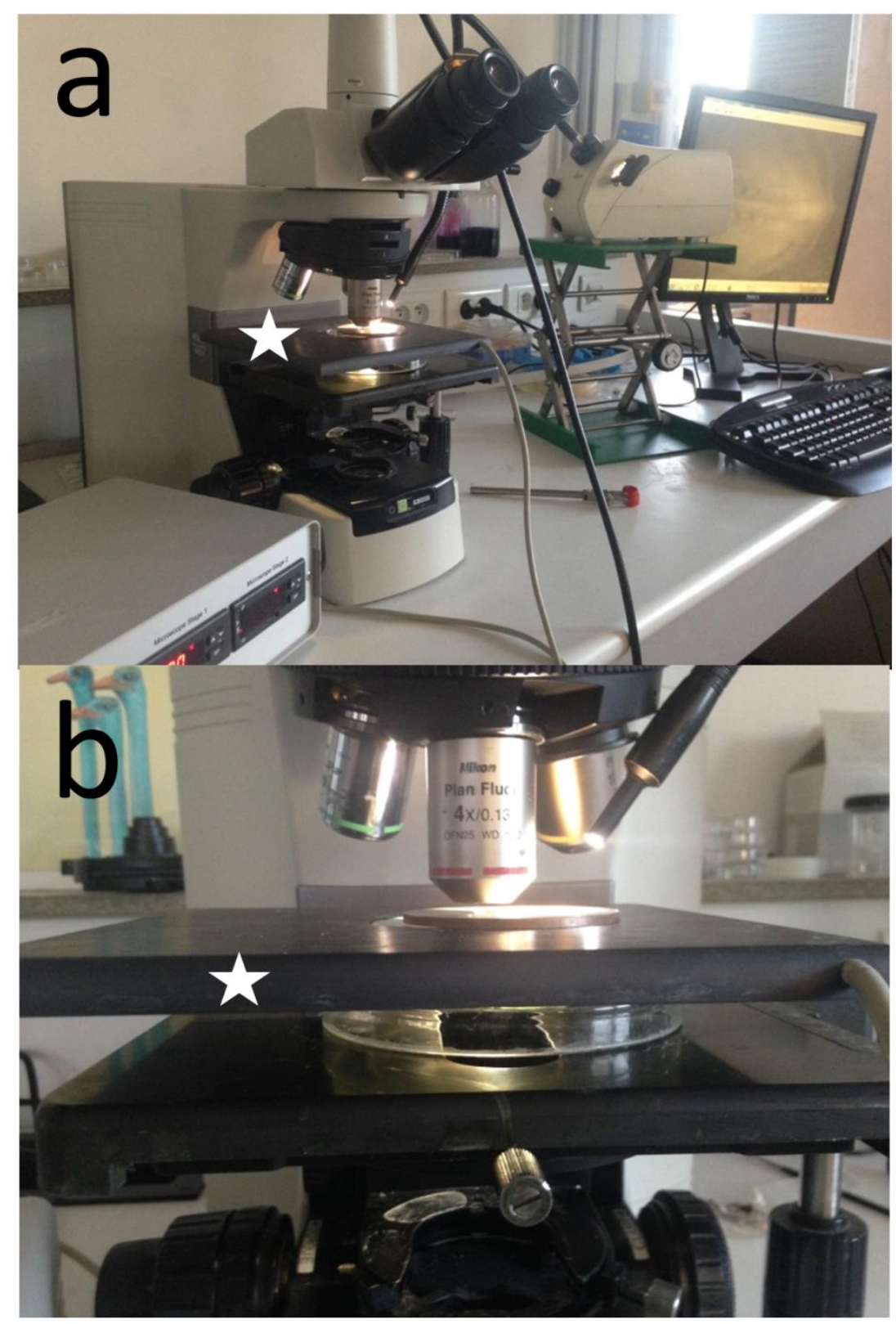

\section{Supplementary Material Figure S5 Title : View of the set-up ready for time-lapse}

fiming. The star shows the Minituib heating stage, deposited on the incubating chamber sealed with the albumin. The dimensions of the incubating chamber are calculated strictly so that the heating stage passes under the Nikon long working distance objectives, and also the two-slits copper disk used to increase the contrasts (visible under the objective in (b) ). (a) shows a wider view, with the temperature controller to the left, and the Schott lamp to the right of the microscope. In the bakcground of (a), the computer screen shows an actual view of the embryo tail bud. 

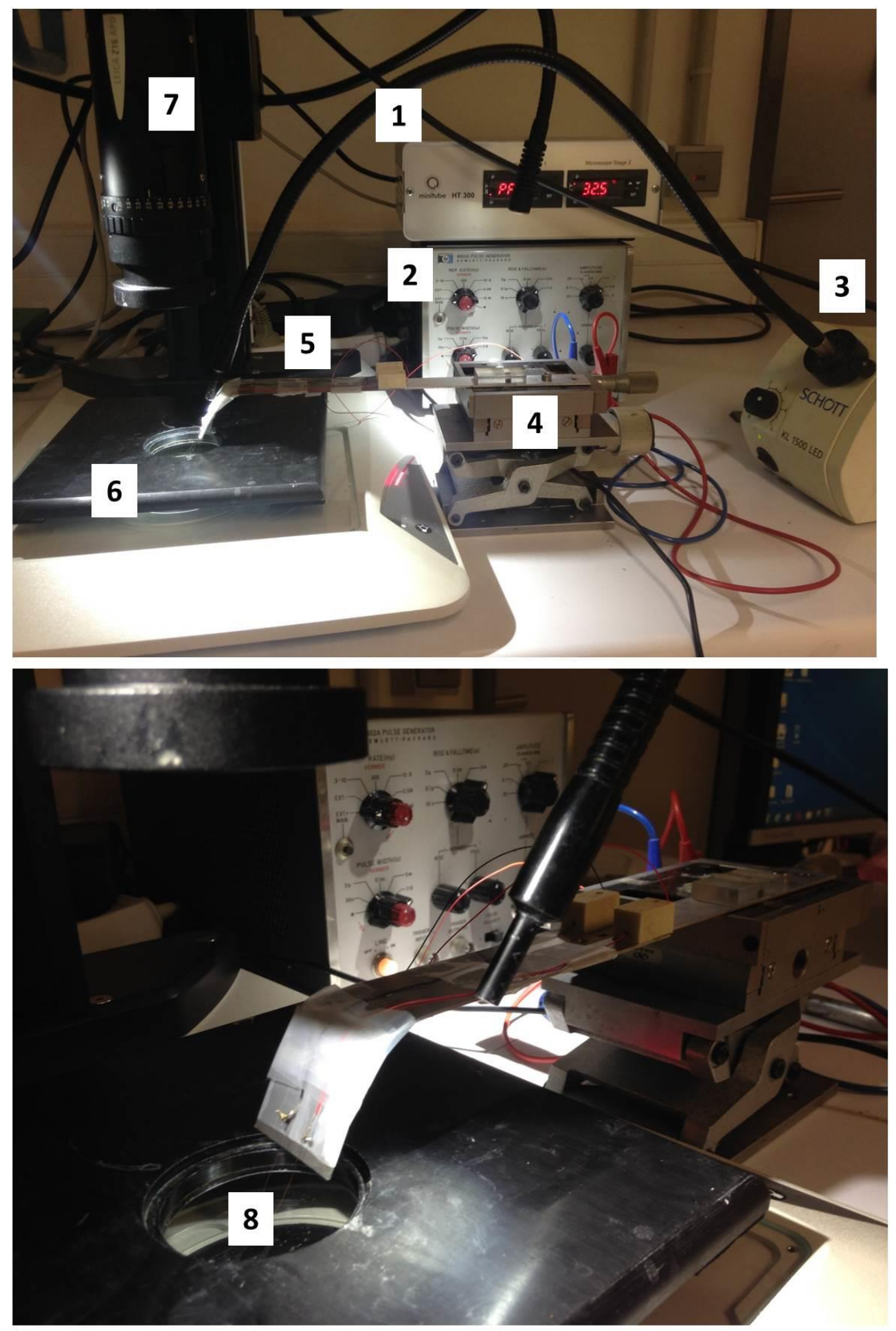

Supplementary Material Figure S6 Title : Set-up for electric shocks. (1) Temperature controller (2) Pulse generator, (3) Fiber-lamp from Schott (4) Micro-positionning stage, (5) Aluminum board used to approach the electrodes, (6) Minitüb heating stage, (7) Leica Macro Fluo objective, (8) incubating chamber opened to pass the electrodes. 


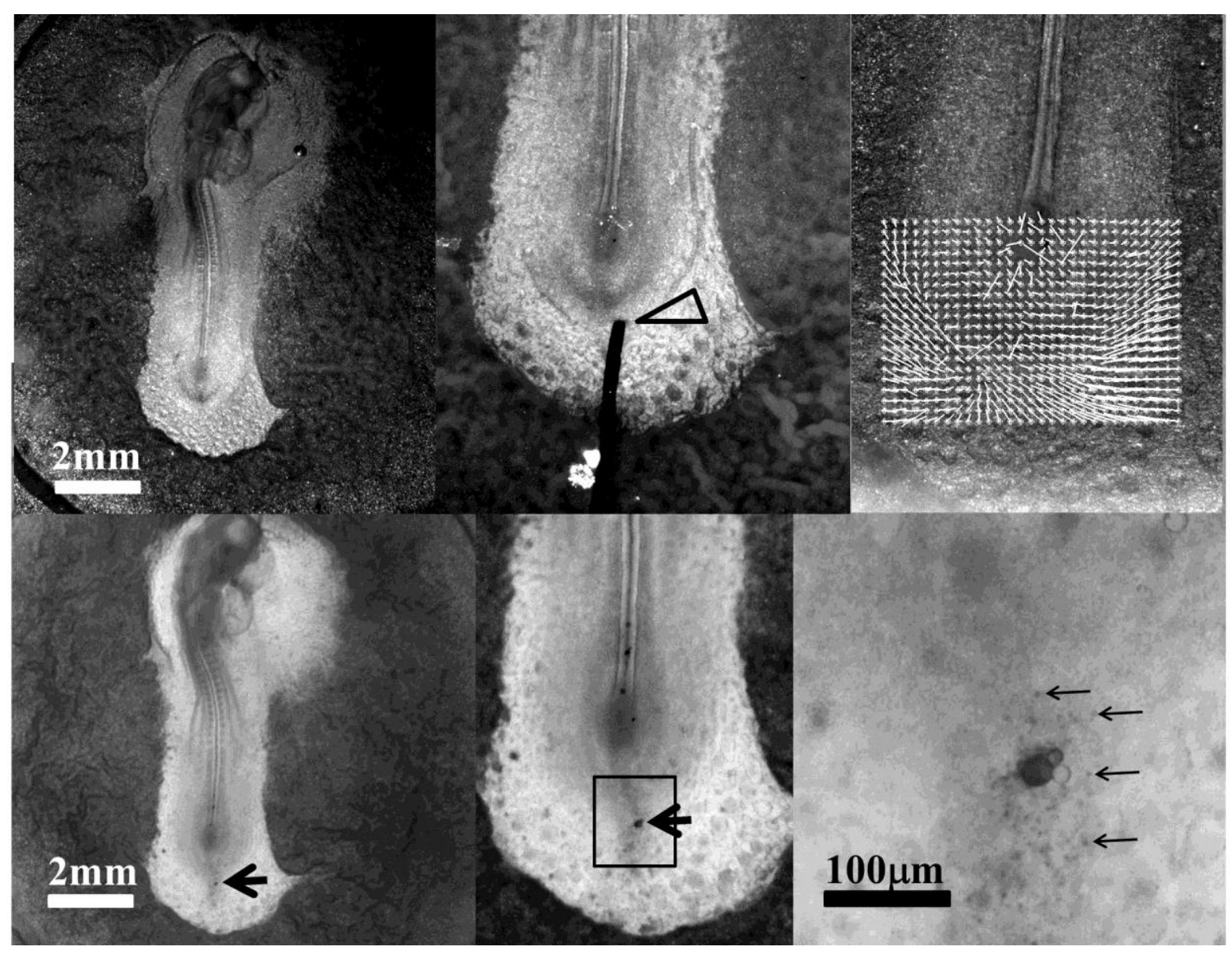

Supplementary Material Figure S7 Title : Trypan staining of the embryo following an

electric shock. Trypan staining reveals dead cells. We stimulated embryos (x 3 samples) in the anal area (arrowhead) with the second electrode positioned away to the left, outside the embryo, on the yolk-sac. The shock consists of $1.5 \mathrm{~V}$ applied for $10 \mathrm{Sec}$. The contraction pattern is shown in Fig. S7 (Top Row, Right), it reveals a strong contraction oriented towards the anal area, and spousing the curved pattern of the limb plates. Figure S7 (Bottom Row) shows the Trypan staining, revealing a very small necrotic zone (black arrows), with a few dead cells below and around the positive electrode. The contraction of this embryo is shown in the Video 23, the presence of a few dead cells does not impede the contraction movement. 
Video 1. Mag. 4X. Time-Lapse video of the rotatory movements generated by the external rings of cells (duration approx. 8hrs). Used for Fig. 1a.

Video 2. Mag. 10X. Time-Lapse video of the ingression of the ectoderm through the primitive streak, and posterior extension. Duration 12hrs. Used for Fig. 1b and 1c. This video was generated by replacing each plate by a superimposition of 20 minutes of flow. This renders a sensation of streamlines flowing.

Video 3. Mag. 4X. Transition from pre-anal to post-anal tail. Duration of the Video 6hrs. Used for Fig. 3a.

Video 4. Mag. 4X. Transition from pre-anal to post-anal tail. Duration of the Video 6hrs. Used for Fig. $3 b$.

Video 5. Mag. 4X. Transition from pre-anal to post-anal tail. Duration of the Video 6hrs. Used for Fig. $3 b$.

Video 6. Mag. 10X. Magnified view of tail compressing the anus. Duration of the Video 4hrs.

Video 7. Mag 4X-10X Magnified view of the tissue between the tail and the amniotic sac "purse string", during tail bud formation, showing the posterior push. Oriented cells are visible around the presumptive limbs territory. To the extreme right, the amniotic "purse string" of active cells. To the left, the tail bud moves and flattens against the anal area.

Video 8. Mag. 4X. Transition from pre-anal to post-anal tail and up to formation of the chorio-amniotic fold. Duration of the Video 8hrs. Used for Fig. 4a.

Video 9. Mag. 4X. Lateral shear by the tail bud, folding of the limb edge, and early formation of the chorio-amniotic fold. Duration of the Video 4hrs. Used for Fig. 4b (Top 4X, Bottom $16 \mathrm{X})$.

Video 10. Mag. 4X. Transition from pre-anal to post-anal tail and up to formation of the Apical Ectodermal Ridge. Duration of the Video 6hrs. Used for Fig. 5a.

Video 11. Mag. 4X. Transition from pre-anal to post-anal tail and up to formation of the Apical Ectodermal Ridge. Duration of the Video 6hrs. Used for Fig. 5b-Top 
Video 12. Mag. 4X. Formation of the limb edge and of the amniotic sac, until the posterior area is entirely wrapped by the amnion. Used for Figs. 5b-Bottom and Fig. 5c. Duration of the Video $15 \mathrm{hrs}$.

Video 13. Low magnificaton Time-Lapse during tail bud formation showing that the posterior movement of the tail area, coincides with an anterior advection of the vertebrate precursors (the somites).

Video 14. Mag. 4X. Reaction of the tissue in the periphery of the presumptive anus during the early gastrulation movements, and following a short ( $3 \mathrm{Sec}$ ) electric shock of amplitude $1.5 \mathrm{~V}$. A rapid contraction of the tissue is observed which amplifies by $20 \mathrm{X}$ the physiological contraction. Duration of the Video 1hr.

Video 15. Mag. 4X. Reaction of the tissue in the periphery of the anus during onset of neurulation while the embryo starts to be pulled posteriorily following a short ( $3 \mathrm{Sec}$ ) electric shock of amplitude $1.5 \mathrm{~V}$. A long rage contraction is obtained. Duration of the Video $1 \mathrm{hr}$.

Video 16. Mag. 3X. Reaction of the tissue in the periphery of the anus during neurulation, and following a short electric shock of amplitude $1.5 \mathrm{~V}$. The tail bud is not yet formed. The electrode is visible in the bottom, in the first plates. The contraction induced in the presumptive anal area is particularly visible. Duration of the Video $1 \mathrm{hr}$.

Video 17. Mag. 3X. Reaction of the tissue in the periphery of the anus during formation of the tail bud, and following a short electric shock of amplitude $1.5 \mathrm{~V}$. The tail bud has not yet passed over the anus, the anal area is already being compressed. One sees the contraction of the tissue around the posterior area, and the artefactual nucleation of an amniotic fold. The electrode is visible just facing the anus, in the first plates. Duration of the Video $2 \mathrm{hrs}$.

Video 18. Mag. 4X. Reaction of the tissue in the periphery of the anus during transition from a pre-anal to a post-anal tail bud, and following a short electric shock of amplitude $1.5 \mathrm{~V}$. The 
electrode is visible just facing the anus, in the first plates. The tail bud is pulled and the tissue constricts following the ring pattern of the tissue. Duration of the Video 2hrs.

Video 19. Mag. 4X. Reaction of the tissue in the lateral plates while the tail bud starts to hang over the anus, following a short electric shock of amplitude $1.5 \mathrm{~V}$ in the anal area. The AER nucleates by the minute and the limb fold starts to propagate. The electrode is visible just facing the anus, in the first plates. Duration of the Video $2 \mathrm{hrs}$.

Video 20. Mag. 4X. Reaction of the embryo following a short electric shock of amplitude $1.5 \mathrm{~V}$ in the periphery of the presumptive limb territory (complete embryo roll up and formation of the amniotic fold). The electrode is visible to the left of the limb edge, in the first plates. Duration of the Video $2 \mathrm{hrs}$.

Video 21 Mag. 4X. Reaction of the amniotic sac following a short electric shock of amplitude $1.5 \mathrm{~V}$ near the edge of the closing amniotic sac. Duration of the Video 25 minutes.

Video 22. Mag. 4X. Reaction of the lateral plate following a short electric shock of amplitude $1.5 \mathrm{~V}$ near the edge of the presumptive limb. Duration of the Video $1 \mathrm{hr}$.

Video 23. Mag. 3X. Reaction of the tissue in the periphery of the anus during formation of the tail bud, and following a short electric shock of amplitude $1.5 \mathrm{~V}$ in the anal area. One sees the contraction of the tissue towards the anus, and the relaxation of the tissue (duration of the Video 1 hour). This Video was used for the Supplementary Material Figure 7, and the Fig. $10 \mathrm{c}$. 